\title{
Superconductivity mediated by a soft phonon mode: Specific heat, resistivity, thermal expansion, and magnetization of $\mathbf{Y B}_{6}$
}

\author{
R. Lortz,,${ }^{1 *}$ Y. Wang, ${ }^{1}$ U. Tutsch, ${ }^{1}$ S. Abe,${ }^{1}$ C. Meingast,${ }^{2}$ P. Popovich,,${ }^{2,3}$ W. Knafo, ${ }^{2,3}$ N. Shitsevalova, ${ }^{4}$ Yu. B. Paderno, ${ }^{4, \dagger}$ \\ and A. Junod ${ }^{1}$ \\ ${ }^{1}$ Department of Condensed Matter Physics, University of Geneva, CH-1211 Geneva 4, Switzerland \\ ${ }^{2}$ Forschungszentrum Karlsruhe, Institut für Festkörperphysik, D-76021 Karlsruhe, Germany \\ ${ }^{3}$ Physikalisches Institut, Universität Karlsruhe, D-76128 Karlsruhe, Germany \\ ${ }^{4}$ Institute for Problems of Materials Science NASU, 03680 Kiev, Ukraine
}

(Received 20 October 2005; published 26 January 2006)

\begin{abstract}
The superconductor $\mathrm{YB}_{6}$ has the second highest critical temperature $T_{c}$ among the boride family $M \mathrm{~B}_{\mathrm{n}}$. We report measurements of the specific heat, resistivity, magnetic susceptibility, and thermal expansion from 2 to $300 \mathrm{~K}$, using a single crystal with $T_{c}=7.2 \mathrm{~K}$. The superconducting gap is characteristic of medium-strong coupling. The specific heat, resistivity, and expansivity curves are deconvolved to yield approximations of the phonon density of states $F(\omega)$, the spectral electron-phonon scattering function $\alpha_{t r}^{2} F(\omega)$, and the phonon density of states weighted by the frequency-dependent Grüneisen parameter $\gamma_{G}(\omega) F(\omega)$, respectively. Lattice vibrations extend to high frequencies $>100 \mathrm{meV}$, but a dominant Einstein-like mode at $\sim 8 \mathrm{meV}$, associated with the vibrations of yttrium ions in oversized boron cages, appears to provide most of the superconducting coupling and gives rise to an unusual temperature behavior of several observable quantities. A surface critical field $H_{\mathrm{c} 3}$ is also observed.
\end{abstract}

DOI: 10.1103/PhysRevB.73.024512

PACS number(s): 74.70.Ad, 74.25.Bt, 72.15.Eb, 65.40.De

\section{INTRODUCTION}

The discovery of superconductivity at $\sim 40 \mathrm{~K}$ in the metallic compound $\mathrm{MgB}_{2}$ (Refs. 1 and 2) has stimulated a renewed interest in borides. The next highest superconducting critical temperatures in the $M \mathrm{~B}_{\mathrm{n}}$ family are found in $\mathrm{YB}_{6}$ with $T_{\mathrm{c}} \leqslant 8.4 \mathrm{~K}$ and $\mathrm{ZrB}_{12}$ with $T_{\mathrm{c}}=6.0 \mathrm{~K}^{3}$ These compounds are hard materials with a low density of states at the Fermi level. Their crystal structure- $\mathrm{CaB}_{6}$ type (bcc, $P m \overline{3} m-O_{h}^{1}$ ) for $\mathrm{YB}_{6}$ and $\mathrm{UB}_{12}$ type (fcc, $F m \overline{3} m-O_{h}^{5}$ ) for $\mathrm{ZrB}_{12}$-is three dimensional and characterized by boron cages in which yttrium or zirconium atoms can develop large vibrational amplitudes. The metal-boron distance $(2.76 \AA$ in $\mathrm{ZrB}_{12}$ and $3.01 \AA$ in $\mathrm{YB}_{6}$ ) is remarkably large, giving rise to unusual properties. $\mathrm{ZrB}_{12}$ was recently investigated with respect to its specific heat, resistivity, thermal expansion, and magnetic properties. ${ }^{4,5}$ Characteristic features of $\mathrm{ZrB}_{12}$ were type-II/1 superconductivity, ${ }^{6}$ an enhanced gap and critical field at the surface, and weak electron-phonon coupling essentially driven by a single anharmonic mode at $14 \mathrm{meV}$. In this work we turn to $\mathrm{YB}_{6}$, the specific heat of which was only briefly mentioned in an early paper, ${ }^{7}$ and find that some of these peculiarities are also present and even more dramatic. Here, a corresponding anharmonic lattice mode has softened to $8 \mathrm{meV}$, giving rise to a very unusual temperature dependence of the specific heat, resistivity, and expansivity. This effect is so marked that $\mathrm{YB}_{6}$ can be considered as a textbook example of superconductivity in an Einstein lattice, a limiting situation where strong-coupling theory is well assessed. ${ }^{8}$ Another consequence of having low-energy modes is that "thermal" spectroscopies-i.e., experiments sensitive to a density of energy states convolved with a thermal distribution-become efficient. In this work we show that specific heat and resistivity can indeed provide information usually taken from inelastic neutron scattering and tunneling spectroscopy, with a limited but sufficient accuracy to characterize the superconducting coupling at a quantitative level.

This article is organized as follows. In Sec. II experimental details and basic sample characterizations are given. The electronic specific heat is analyzed in Sec. III. Sections IV-VI are devoted to the deconvolution of the specific heat, resistivity, and thermal expansivity curves to obtain information on the phonon density of states $F(\omega)$, electron-phonon transport coupling function $\alpha_{t r}^{2} F(\omega)$, and frequencydependent Grüneisen parameter $\gamma_{G}(\omega)$, respectively. The magnetic phase diagram with four critical field lines is established in Sec. VII, based on different experiments. The compositional dependence is briefly addressed in Sec. VIII, before concluding in Sec. IX. A wealth of experimental information is already available for $\mathrm{YB}_{6}$ in the literature, and we refer to Ref. 9 for a review.

\section{CRYSTAL GROWTH AND EXPERIMENTAL DETAILS}

The preparation of single crystals of yttrium hexaboride involved several steps: synthesis of $\mathrm{YB}_{6}$ powder by borothermal reduction of $\mathrm{Y}_{2} \mathrm{O}_{3}$, compacting the powder into rods, sintering, and zone melting by inductive heating. Since the superconducting critical temperature of $\mathrm{YB}_{6}$ is particularly sensitive to impurities, 99.999\%-pure $\mathrm{Y}_{2} \mathrm{O}_{3}$ powder and $>99.5 \%$ amorphous boron were used as starting materials. The highly volatile impurities contained in the latter were eliminated during the synthesis and zone melting procedures, resulting in a total impurity concentration in the crystals under study of at most $0.001 \%$ in weight.

Taking into account the peritectic melting of $\mathrm{YB}_{6}$ (Ref. 10) and previous results on crystal growth, ${ }^{11}$ we synthesized 

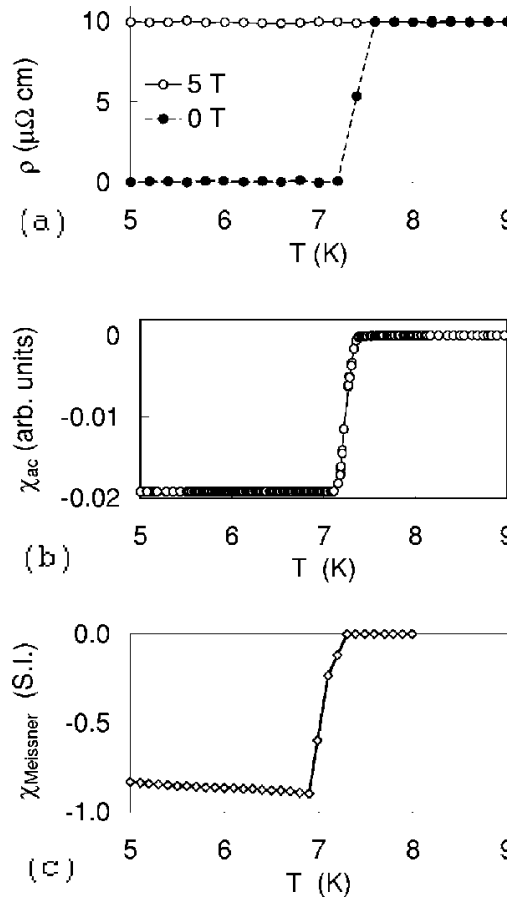

FIG. 1. Superconducting transition of the $\mathrm{YB}_{6}$ crystal observed by (a) resistivity, (b) ac susceptibility ( $8 \mathrm{kHz}, 0.01 \mathrm{G} \mathrm{rms}$ ), and (c) Meissner susceptibility (field cooled, $2.7 \mathrm{G}$ ).

the initial powders with excess boron in order to lower the melting temperature. High-quality single crystals were obtained for a source composition $\mathrm{B} / \mathrm{Y}>6.8$. Other important technological parameters were the pressure of argon gas, 1.3 $\mathrm{MPa}$, and the growth rate, $13 \mathrm{~mm} / \mathrm{h}$. The growth was unstable over the first few mm, yielding a two-phase mixture of $\mathrm{YB}_{4}$ and $\mathrm{YB}_{6}$. The process subsequently stabilized and at a definite B/Y ratio a single-phase ingot started to grow. Due to concurrent growth, one grain grew at the expense of the others and approximately $\langle 100\rangle$-oriented single crystals were obtained with a length of about $30 \mathrm{~mm}$.

The as-grown single crystal used for nearly all subsequent experiments was shaped into a parallelepiped bar by spark cutting and then polished using abrasive diamond paste. Its dimensions are $\sim 12 \times 3 \times 1.5 \mathrm{~mm}^{3}$ and mass $73.5 \mathrm{mg}$, with the long axis parallel to the $\langle 100\rangle$ direction and the facets perpendicular to $\langle 010\rangle$ and $\langle 001\rangle$. Measurements in a magnetic field were taken with the field along the length of the sample in order to minimize the demagnetization factor $(D$ $\cong 0.03$ ). The superconducting transition temperature $T_{c}$ was determined by four methods: resistivity [Fig. 1(a)], ac susceptibility [Fig. 1(b)], dc magnetization [Fig. 1(c)], and specific-heat jump at $T_{c}$ (see Fig. 3 below) which, in this order, are increasingly representative of the bulk volume. On average, $T_{c} \cong 7.2 \mathrm{~K}$, the transition width being $\sim 2 \%$ of $T_{c}$ (Table I).

The dc resistivity $\rho$ was measured by a four-lead technique with current reversal, using a $5 \mathrm{~mA}$ current. The contacts were made with Degussa "Leitsilber 200" conducting silver paint. The residual resistivity ratio was $\rho(300 \mathrm{~K}) / \rho(2 \mathrm{~K})=3.87$. The residual resistivity $\rho(2 \mathrm{~K})$ $=9.9 \mu \Omega \mathrm{cm}$ was determined in a magnetic field of $5 \mathrm{~T}$ to
TABLE I. Critical temperature and transition width of the $\mathrm{YB}_{6}$ crystal measured by different methods.

\begin{tabular}{lcc}
\hline \hline & $T_{c}$ midpoint $(\mathrm{K})$ & $\Delta T_{c}(\mathrm{~K})$ \\
\hline $\begin{array}{l}\text { Resistivity in zero field } \\
\text { at } 1 \mathrm{~mA} / \mathrm{mm}^{2}\end{array}$ & 7.20 & $<0.2(0 \%-100 \%)$ \\
$\begin{array}{l}\text { ac susceptibility at } 8 \mathrm{kHz}, \\
0.01 \text { Oe }\end{array}$ & 7.24 & $0.15(10 \%-90 \%)$ \\
$\begin{array}{l}\text { Meissner magnetization } \\
\text { at } 2.7 \text { Oe }\end{array}$ & 7.13 & $0.20(10 \%-90 \%)$ \\
$\begin{array}{l}\text { Specific heat jump } \\
\text { in zero field }\end{array}$ & 7.15 & $0.13(10 \%-90 \%)$ \\
\hline \hline
\end{tabular}

quench superconductivity; the resistivity did not vary appreciably below $9 \mathrm{~K}$. No significant magnetoresistance was observed in the normal state.

The magnetization $M$ was measured in a Quantum Design MPMS-5 magnetometer, using a scan length of $4 \mathrm{~cm}$. The zero-field-cooled (ZFC) susceptibility in the Meissner state was measured in a field of $2.7 \mathrm{Oe}$, which is $\sim 1 \%$ of the lower critical field at $T=0$ [Fig. 1(c)]. The normal-state susceptibility $\chi(T)$ was obtained from the initial slope of $M(H)$ measured at 1-T intervals from 0 to $5 \mathrm{~T}$. The core and Pauli contributions nearly cancel each other, resulting in a small and slightly diamagnetic susceptibility. A Curie component develops at low temperature, possibly due to traces of magnetic rare earth metals in the Y starting material (Fig. 2). The best fit is obtained by allowing a second-order term in the Pauli susceptibility:

$$
\chi(T)=\chi(0)+a T^{2}+\frac{C_{\text {Curie }}}{T} .
$$

The fitted parameters are (S.I.) $\chi(0)=-9.6 \times 10^{-6}, a=2.4$ $\times 10^{-11} \mathrm{~K}^{-2}$, and $C_{\text {Curie }}=1.8 \times 10^{-4} \mathrm{~K}$. The Curie term is equivalent to $35 \mathrm{ppm}$ at. $\mathrm{Yb}^{3+}$.

The specific heat was measured by a generalized relaxation technique at low temperature (1.2-15 K) (Ref. 12) and in an adiabatic, continuous-heating calorimeter at high temperature $(16-300 \mathrm{~K}){ }^{12}$ Care was taken to measure the re-

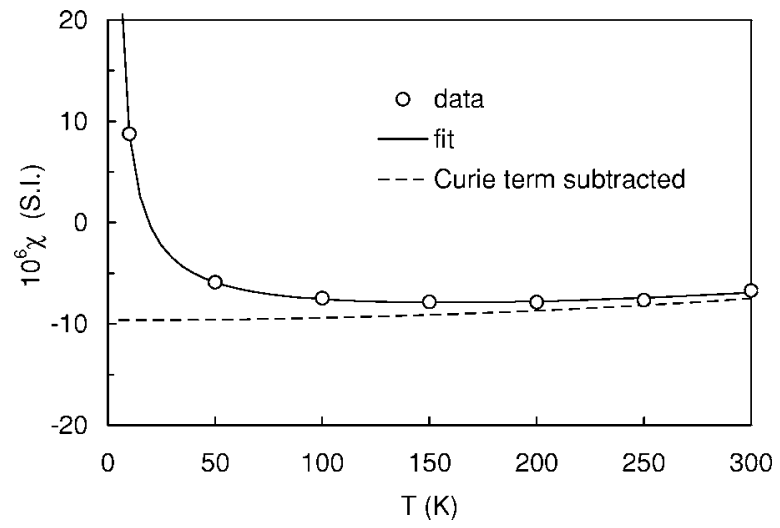

FIG. 2. Magnetic susceptibility of $\mathrm{YB}_{6}$ in the normal state as a function of the temperature. Solid line: fit (see text). Dashed line: non-Curie part of the fit. 


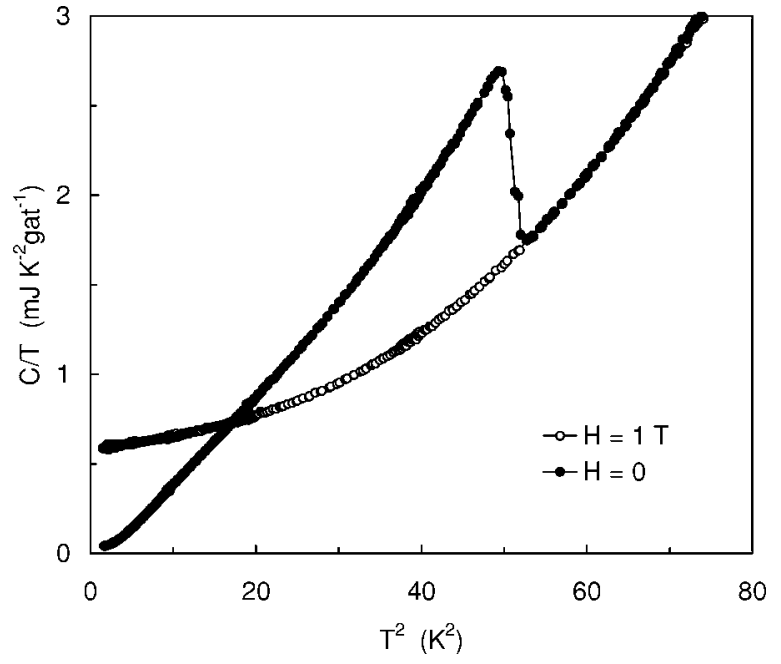

FIG. 3. Total specific heat $C / T$ of $\mathrm{YB}_{6}$ in the superconducting state in zero field (solid symbols) and in the normal state in $1 \mathrm{~T}$ (open symbols) vs the temperature squared.

sidual field of the $14 \mathrm{~T}$ magnet mounted in the cryostat, compensating this when required. Specific heat runs were taken after cooling in a field from above $T_{c}$ in order to achieve the best possible field penetration.

A high-resolution capacitance dilatometer ${ }^{13,14}$ was used to measure the thermal expansion in the temperature range $3-250 \mathrm{~K}$. Data were taken both upon heating and cooling at a constant rate of $5 \mathrm{mK} / \mathrm{s}$ at low temperatures $(3-15 \mathrm{~K})$ and at $20 \mathrm{mK} / \mathrm{s}$ up to $250 \mathrm{~K}$. Flowing $\mathrm{He}$ exchange gas (0-4 mbar) was used to thermally couple the sample to the dilatometer.

\section{ELECTRON-SPECIFIC HEAT, DENSITY OF STATES, AND COUPLING STRENGTH}

The specific heat at low temperature in both the superconducting $(H=0)$ and normal state $(H=1 \mathrm{~T})$ is shown in Fig. 3. The superconducting-state specific heat $C_{S}$ shows a sharp second-order jump at $T_{c}$. It vanishes at $T \rightarrow 0$ in a somewhat unusual way, since $C_{s} / T$ tends to a finite value $\gamma_{0}$ $=0.03 \mathrm{~mJ} \mathrm{~K}^{-2} \mathrm{gat}^{-1}$ rather than zero as would be expected in a fully gapped state (one gram-atom [gat] is one Avogadro's number of atoms, here one-seventh of a mole (Table II). This residual contribution, which may be due to an extrinsic nonsuperconducting fraction or to a gapless electron band, will not be discussed further as it only represents $\sim 5 \%$ of the normal-state electronic specific heat. The normal-state specific heat is analyzed in a standard way according to the expansion

$$
C_{n}(T \rightarrow 0)=\gamma_{n} T+\sum_{n=1}^{3} \beta_{2 n+1} T^{2 n+1},
$$

where the first term is the electronic contribution, with $\gamma_{n}$ $=\frac{1}{3} \pi^{2} k_{B}^{2}\left(1+\lambda_{e p}\right) N\left(E_{F}\right), \quad k_{B}$ Boltzmann's constant, $\lambda_{e p}$ the electron-phonon coupling constant and $N\left(E_{F}\right)$ the bandstructure density of states at the Fermi level including two
TABLE II. Characteristic parameters of $\mathrm{YB}_{6}$ compared to $\mathrm{ZrB}_{12} . T_{c}$, superconducting transition temperature; RRR, residual resistivity ratio; $V$ and $M$, mean atomic volume and mass, respectively; $a$, lattice constant; $\gamma_{n}$, coefficient of the linear term of the normal-state specific heat at $T \rightarrow 0 ; \Delta C / T_{c}$, specific heat jump at $T_{c}$; $\Delta C / \gamma_{n} T_{c}$, normalized specific heat jump; $\Theta_{D}(0)$, initial Debye temperature; $S(300)$, total entropy at $300 \mathrm{~K} ; E_{c}$, condensation energy at $T \rightarrow 0 ; 2 \Delta(0) / k_{B} T_{c}$, normalized superconducting gap; $\chi(0)$, normalstate magnetic susceptibility at $T \rightarrow 0 ; N_{s b}\left(E_{F}\right)$, bare density of states at the Fermi level [per 7-atom cell for $\mathrm{YB}_{6}$ (Ref. 19-21), per 13-atom quarter-cell for $\mathrm{ZrB}_{12}$ (Refs. 21 and 55)]; $1+\lambda_{\text {ep }}$, electronphonon renormalization factor as determined from $\gamma_{n}$ and $N_{s b}\left(E_{F}\right)$; $\lambda_{e p}$, electron-phonon coupling constant as determined from $T_{c}$ and $\omega_{\ln }$ (Ref. 22).

\begin{tabular}{|c|c|c|}
\hline & $\mathrm{YB}_{6}$ & $\mathrm{ZrB}_{12}$ \\
\hline$T_{c}(\mathrm{~K})$ & $7.15 \pm 0.05$ & $5.96 \pm 0.05$ \\
\hline RRR & $3.87 \pm 0.03$ & $9.33 \pm 0.03$ \\
\hline$V\left(\mathrm{~cm}^{3} \mathrm{gat}^{-1}\right)$ & 5.929 & 4.68 \\
\hline$M\left(\mathrm{~g} \mathrm{gat}^{-1}\right)$ & 21.97 & 17.0 \\
\hline$a(\mathrm{~nm})$ & $0.41002 \pm 0.00005$ & 0.7407 \\
\hline$\gamma_{n}\left(\mathrm{~mJ} \mathrm{~K}^{-2} \mathrm{gat}^{-1}\right)$ & $0.58 \pm 0.02$ & $0.34 \pm 0.02$ \\
\hline$\Delta C / T_{c}\left(\mathrm{~mJ} \mathrm{~K}^{-2} \mathrm{gat}^{-1}\right)$ & $1.18 \pm 0.02$ & $0.56 \pm 0.02$ \\
\hline$\Delta C / \gamma_{n} T_{c}$ & $2.02 \pm 0.1$ & $1.66 \pm 0.1$ \\
\hline$\theta_{D}(0)(\mathrm{K})$ & $706 \pm 20$ & $970 \pm 20$ \\
\hline$S(300)\left(\mathrm{J} \mathrm{K}^{-1} \mathrm{gat}^{-1}\right)$ & $13.5 \pm 0.1$ & $9.3 \pm 0.1$ \\
\hline$E_{c}(\mathrm{~mJ} / \mathrm{gat})$ & $7.15 \pm 0.2$ & $3.2 \pm 0.1$ \\
\hline $2 \Delta(0) / k_{B} T_{c}$ & $4.1 \pm 0.1$ & $3.7 \pm 0.1$ \\
\hline$\chi(0)($ S.I. $)$ & $-9.6 \times 10^{-6}$ & $-20.8 \times 10^{-6}$ \\
\hline$N_{s b}\left(E_{F}\right)(\mathrm{eV} \text { cell })^{-1}$ & $0.81-0.93$ & $1.57-1.59$ \\
\hline $1+\lambda_{e p}\left(\right.$ from $\left.\gamma_{n}\right)$ & $1.86-2.14$ & $1.18-1.19$ \\
\hline$\lambda_{e p}\left(\right.$ from $\left.T_{c}\right)$ & 1.01 & $0.61-0.65$ \\
\hline
\end{tabular}

spin directions [electronic density of states (EDOS)]. The second term is the low-temperature expansion of the lattice specific heat, where $\beta_{3}=\frac{12}{5} N_{A v} k_{B} \pi^{4} \theta_{D}^{-3}(0)$, with $N_{A v}$ Avogadro's number and $\theta_{D}(0)$ the initial Debye temperature. From a fit of the normal-state data from 1.2 to $7.5 \mathrm{~K}$, we obtain $\gamma_{n}=0.58 \mathrm{~mJ} \mathrm{~K}^{-2} \mathrm{gat}^{-1}$ and $\theta_{D}(0)=706 \mathrm{~K}(369 \mathrm{~K}$ if according to another convention only acoustic modes are considered). The Sommerfeld constant $\gamma_{n}$, which is $\sim 50 \%$ larger than that mentioned by Matthias et al., ${ }^{7}$ corresponds to a renormalized EDOS $\left(1+\lambda_{e p}\right) N\left(E_{F}\right)=1.73 \mathrm{eV}^{-1}$ cell $^{-1}$. The band structure has been calculated by several groups. ${ }^{15-21}$ Comparing the renormalized EDOS with the recently obtained values $N\left(E_{F}\right)=0.82$ (Ref. 19), 0.83 (Ref. 20), and 0.93 (Ref. 21) $\mathrm{eV}^{-1}$ cell $^{-1}$, we find room for an electron-phonon renormalization factor $\approx 2$-i.e., $\lambda_{e p}=1.12,1.08$, and 0.86 , respectively. The value $\lambda_{e p} \approx 1$ is supported by independent determinations. For example, we may start from $\Delta C / \gamma_{n} T_{c}$, the normalized specific heat jump at $T_{c}$, as a well-defined input observable. From there we evaluate $T_{c} / \omega_{\mathrm{ln}}=0.071$ using isotropic single-band strong-coupling formulas representing interpolated solutions of Eliashberg equations (Eq. 5.9 of Ref. 8; $\omega_{\mathrm{ln}}$ is the logarithmic moment $\exp \left[\int \ln \omega \alpha^{2} F(\omega) d \omega / \omega / \int \alpha^{2} F(\omega) d \omega / \omega\right]$ of the Eliashberg 
TABLE III. $H_{c}(0)$, thermodynamic critical field at $T \rightarrow 0$ obtained from specific heat $(C)$ and magnetization $(M)$ measurements; $\left(d H_{c} / d T\right)_{T_{c}}$, slope of the thermodynamic critical field at $T \rightarrow T_{c}$; $H_{c 1}(0)$, lower critical field at $T \rightarrow 0 ; H_{c 2}(0)$, upper critical field at $T \rightarrow 0 ;\left(d H_{c 2} / d T\right)_{T c}$, slope of the upper critical field at $T \rightarrow T_{c}$; $H_{c 3}(0)$, surface upper critical field at $T \rightarrow 0 ; \kappa \equiv \kappa_{1}\left(T_{c}\right)$, Maki parameter.

\begin{tabular}{ll}
$H_{c}(0)(\mathrm{mT})$ & $55(C), 58(M)$ \\
$\left(d H_{c} / d T\right)_{T_{c}}(\mathrm{mT} / \mathrm{K})$ & -15.7 \\
$H_{c 1}(0)(\mathrm{mT})$ & $20-25$ \\
$H_{c 2}(0)(\mathrm{mT})$ & 295 \\
$\left(d H_{c 2} / d T\right)_{T_{c}}(\mathrm{mT} / \mathrm{K})$ & -59 \\
$H_{c 3}(0)(\mathrm{mT})$ & $\sim 640$ \\
$\kappa_{1}\left(T_{c}\right)$ & 3.0 \\
\hline
\end{tabular}

function $\alpha^{2} F(\omega)$ ). Assuming the conventional value $\mu^{*}$ $=0.10$ for the Coulomb pseudopotential, ${ }^{22}$ we obtain $\lambda_{e p}$ $=1.01$ according to the Allen-Dynes equation. ${ }^{22}$ A change of \pm 0.05 in $\mu^{*}$ affects the value of $\lambda_{e p}$ by \pm 0.15 . In Sec. V we give a third independent determination $\lambda_{e p}=1.04$ which relies on the electrical and optical conductivity and confirms the first two calculations. At this point we already wish to draw attention to the low value $\omega_{\ln }=101 \mathrm{~K} \ll \theta_{D}(0)=706 \mathrm{~K}$ that results from this analysis. $\mathrm{YB}_{6}$ is characterized by selective electron-phonon coupling to low-frequency modes.

The thermodynamic critical field $H_{c}(T)$ is obtained by numerical integration of the specific heat data (Table III):

$$
\begin{aligned}
& -\frac{1}{2} \mu_{0} V H_{c}^{2}(T)=\Delta U(T)-T \Delta S(T), \\
& \Delta U(T)=\int_{T}^{T_{c}}\left[C_{s}\left(T^{\prime}\right)-C_{n}\left(T^{\prime}\right)\right] d T^{\prime}, \\
& \Delta S(T)=\int_{T}^{T_{c}} \frac{C_{S}\left(T^{\prime}\right)-C_{n}\left(T^{\prime}\right)}{T^{\prime}} d T^{\prime},
\end{aligned}
$$

where the volume $V$ and other specific quantities refer to $1 \mathrm{~g}$ at. $H_{c}(T)$ is nearly parabolic and extrapolates to $55 \mathrm{mT}$ at $T=0$ (see Fig. 16, below). The deviation function $D(t) \equiv h$ $-\left(1-t^{2}\right)$, where $h \equiv H_{c}(T) / H_{c}(0)$ and $t \equiv T / T_{c}$, is a good indicator of the coupling strength. ${ }^{8}$ The curve for $\mathrm{YB}_{6}$ (Fig. 4, inset) is very similar to that of $\mathrm{Nb}_{77} \mathrm{Zr}_{23}$, for which $2 \Delta(0) / k_{B} T_{c} \cong 4.2,{ }^{23}$ and is bounded by those of $\mathrm{Nb}$ and $\mathrm{Hg}$, for which $2 \Delta(0) / k_{B} T_{c} \cong 4.0$ and 4.6 , respectively. ${ }^{8}$ Other estimations of the gap ratio rely on the slope of the BCS semilogarithmic plot (Fig. 4), ${ }^{24}$ the normalized specific heat jump at $T_{c}$ [Eq. (4.1) of Ref. 8], or a fit of the $\alpha$ model. ${ }^{25}$ These determinations consistently yield $2 \Delta(0) / k_{B} T_{c}=4.1 \pm 0.1$ and only differ by the weighting given to different temperature ranges. Finally we recall tunneling measurements giving $2 \Delta(0) / k_{B} T_{c}=4.02$ and $\lambda_{e p}=0.90\left(\right.$ with $\left.\mu^{*}=0\right) .{ }^{26}$ All these determinations provide evidence for medium- to strongcoupling superconductivity in $\mathrm{YB}_{6}$. In this respect, $\mathrm{YB}_{6}$ dif-

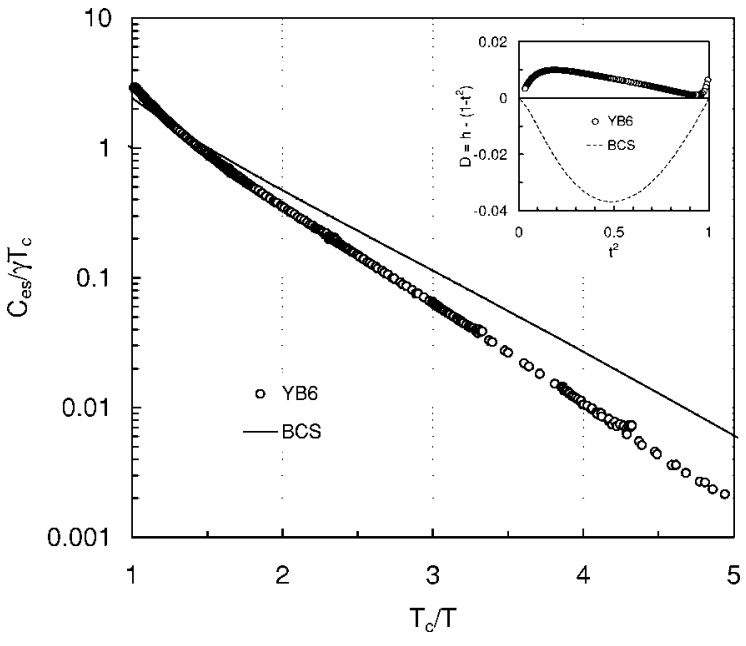

FIG. 4. BCS plot of the electronic specific heat in the superconducting state normalized by the Sommerfeld constant $\gamma_{n}$, vs the inverse reduced temperature, data (symbols), and BCS weakcoupling limit (line). The residual contribution $\gamma_{0} T$ has been subtracted. Inset: deviation function of the thermodynamic critical field (symbols) and BCS weak-coupling limit (dashed line).

fers from $\mathrm{ZrB}_{12}$ which is a weakly coupled superconductor (Table II). ${ }^{4,5}$

Finally, note that the shape of the electronic specific heat in the superconducting state excludes $d$-wave superconductivity. In particular, in the latter case the dimensionless ratio $\gamma_{n} T_{c}^{2} / \mu_{0} V H_{c}^{2}(0)$ would be nearly twice as large (3.7) as that observed $(2.00 \pm 0.05){ }^{27}$

\section{LATTICE SPECIFIC HEAT AND PHONON DENSITY OF STATES}

The low-temperature $T^{3}$ regime of the lattice specific heat does not extend beyond a few kelvin, as shown by the large positive curvature of the normal-state curve in Fig. 3. Huge deviations with respect to the ideal Debye model persist at higher temperature (Fig. 5). The shape of the lattice specific heat in the $C / T$ plot is very uncommon, exhibiting a large low-temperature peak (Fig. 6). The specific heat at room temperature reaches only $\sim 56 \%$ of the Dulong-Petit value, showing that the thermal energy at $300 \mathrm{~K}$ is still too low to excite all the spectral modes. The effective Debye temperature $\theta_{\text {eff }}(T)$ is defined as the value of $\theta$ necessary to fit the experimental specific heat at any $T$ with the equation

$$
C_{p h}(T)=9 N_{A v} k_{B}\left(\frac{T}{\theta}\right)^{3} \int_{0}^{\theta / T} \frac{x^{4} e^{x}}{\left(e^{x}-1\right)^{2}} d x .
$$

We have assumed $C_{p h}=C_{\text {total }}-\gamma_{n} T$, neglecting the anharmonic specific heat. This is justified by the estimation $C_{p}$ $-C_{v}=(3 \alpha)^{2} B V T$, where $B \cong 190 \mathrm{GPa}$ is the bulk modulus and $\alpha \cong 6.1 \times 10^{-6} \mathrm{~K}^{-1}$, the coefficient of linear thermal expansion (see Sec. VI); $\left(C_{p}-C_{v}\right) / C_{p} \cong 0.8 \%$ at $250 \mathrm{~K}$. The electronic term $\gamma_{n} T$ only represents $\sim 1 \%$ of $C_{\text {total }}$ at room temperature. Starting from its initial value $\theta_{\text {eff }}(0)=\theta_{D}(0)$ $=706 \mathrm{~K}$, the effective Debye temperature passes through a 


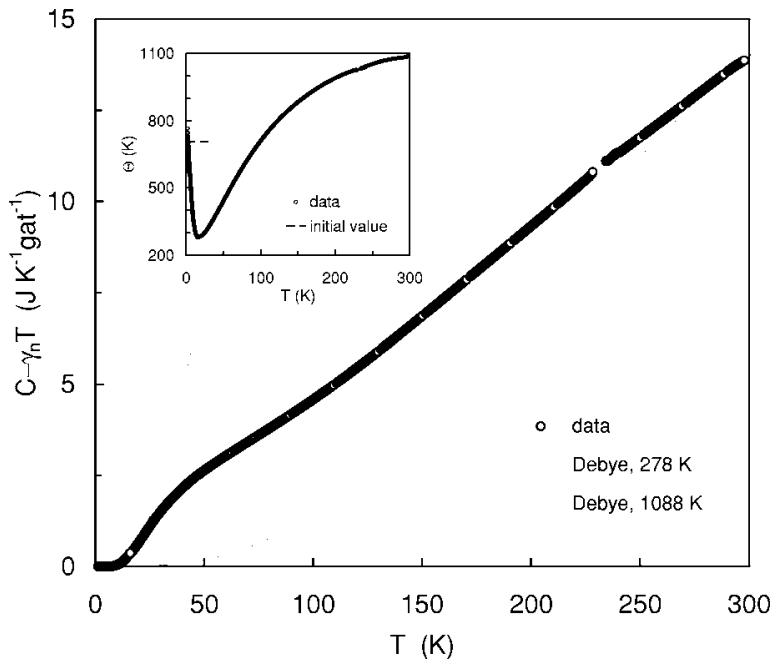

FIG. 5. Lattice-specific heat of $\mathrm{YB}_{6}$ vs the temperature up to room temperature. The long-dashed line shows the Debye-specific heat calculated using a constant Debye temperature equal to its minimum $\theta_{D}(16 \mathrm{~K})=278 \mathrm{~K}$, the short-dashed line using the effective value at room temperature $\theta_{D}(300 \mathrm{~K})=1088 \mathrm{~K}$. Inset: effective Debye temperature vs temperature.

deep minimum $\theta_{\text {eff }}(16 \mathrm{~K})=278 \mathrm{~K}$, then increases monotonically, suggesting an asymptotic value between 1100 and $1200 \mathrm{~K}$. Its room-temperature value is $\theta_{\text {eff }}(300 \mathrm{~K})=1088 \mathrm{~K}$. Figure 5 shows the ideal Debye specific heat for selected values of the Debye temperature. It is clear that the data do not match any curve with a constant $\theta_{D}$. These plots point towards a large phonon density of states (PDOS) at energies of the order of $80-100 \mathrm{~K}$. Analogous situations occuralbeit to a lesser extent-in $\mathrm{Na}$ and $\mathrm{Al}$ due to the presence of optical phonons ${ }^{28}$ and in the borides $\mathrm{ZrB}_{12}$ (Ref. 4) and $\mathrm{LaB}_{6}$ (Ref. 29), for example. The specific-heat data at high temperature are sufficiently minimally scattered to attempt a deconvolution of $C_{p h}(T)$ to extract the PDOS $F(\omega)$. More pre-

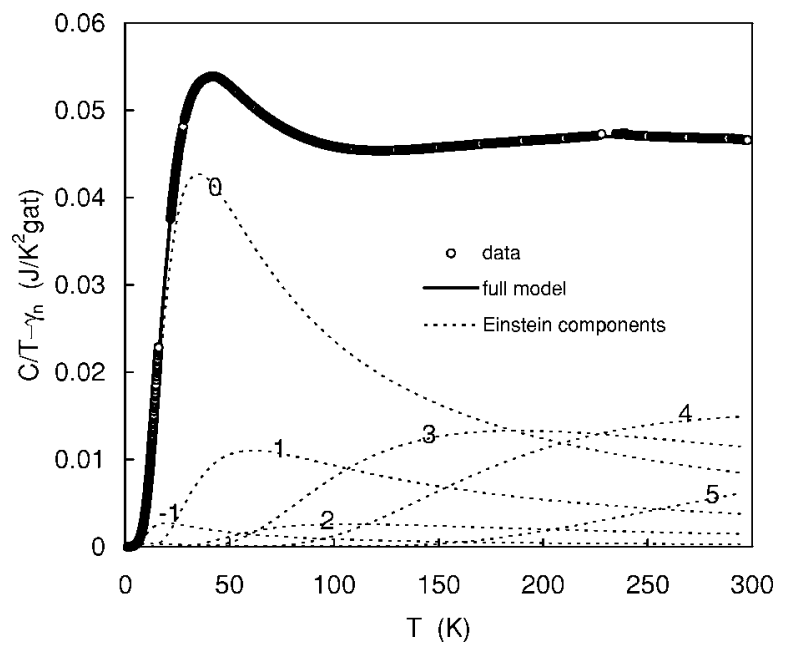

FIG. 6. Lattice-specific heat divided by the temperature vs the temperature showing the decomposition into Einstein terms. The labels $k$ correspond to Einstein temperatures $\Theta_{E, k}=90 \mathrm{~K}$ $\times 1.75^{k}$-i.e. (from left to right), 51, 90, 158, 276, 482, 844, and $1477 \mathrm{~K}$. cisely, we can only obtain a substitutional spectrum-i.e. a smoothed phonon density of states $\bar{F}(\omega)$ which precisely reproduces the specific heat and low-order moments of $F(\omega)$ but may not show the true PDOS in detail. A simplified method consists of representing $F(\omega)$ by a basis of Einstein modes with constant spacing on a logarithmic frequency axis:

$$
F(\omega)=\sum_{k} F_{k} \delta\left(\omega-\omega_{k}\right)
$$

The corresponding lattice specific heat is given by

$$
C_{p h}(T)=3 N_{A v} k_{B} \sum_{k} F_{k} \frac{x_{k}^{2} e^{x_{k}}}{\left(e^{x_{k}}-1\right)^{2}},
$$

where $x_{k}=\omega_{k} / T$. The weights $F_{k}$ are found by a least-squares fit of the lattice specific heat. The number of modes is chosen to be small enough to ensure the stability of the solution; a practical choice is $\omega_{k+1} / \omega_{k}=1.75$. Note that we do not try to find the energy of each mode; we aim rather at establishing a histogram of the density in predefined frequency bins. The robustness of the fit ( $\mathrm{rms}$ deviation $<0.2 \%$ above $16 \mathrm{~K}$ ) is demonstrated by the reproducibility of the results of two independent runs over slightly different temperature ranges (Table IV). The sum of weights exceeds the ideal value 1 by $10 \%$; most probably part of the fitted weight in the highestenergy modes in this harmonic model only serves to simulate the anharmonic contribution. Table IV also gives the generalized moments

$$
\begin{gathered}
\bar{\omega}_{\ln } \equiv \exp \left(\frac{\int \omega^{-1} \ln \omega F(\omega) d \omega}{\int \omega^{-1} F(\omega) d \omega}\right), \\
\left\langle\bar{\omega}^{2}\right\rangle^{1 / 2} \equiv\left(\frac{\int \omega F(\omega) d \omega}{\int \omega^{-1} F(\omega) d \omega}\right)^{1 / 2},
\end{gathered}
$$

to be compared later with similar moments of the Eliashberg function $\alpha^{2} F(\omega)$. Figure 6 illustrates the decomposition of the lattice-specific heat into Einstein contributions. The PDOS obtained in this way is shown in Fig. 9 below. It consists of a background with a high cutoff frequency, as expected in view of the light and rigid boron sublattice, superposed onto a strong peak at $\sim 8 \mathrm{meV}$ which is associated with nearly free oscillations of the $\mathrm{Y}$ atoms in oversized boron cages. The relative weight of the latter peak, $\sim 10 \%$ ( $\sim 15 \%$ if we include both neighboring energy bins), is of the order of the fraction of $\mathrm{Y}$ atoms per formula unit. The question therefore arises as to what extent this low-energy region of the PDOS contributes to the electron-phonon coupling. Different answers have been given in the literature, with the main coupling being attributed to either the boron sublattice $^{16}$ or translational modes involving the yttrium ions. ${ }^{26}$ This point is addressed in the next section, using resistivity as an experimental probe. 
TABLE IV. Results of the fit of the specific heat in terms of $F(\omega)$ and the fit of the resistivity in terms of $\alpha^{2} F_{t r}(\omega) . \omega_{k}$, energy of the modes in a geometrical series $\omega_{k+1}=1.75 \omega_{k}$. The origin is arbitrarily chosen. $F_{k}$, weight associated with each mode in $F(\omega)$; two sets of numbers show the results of two independent runs. A $(-)$ sign means that this mode was not included in the fit. $\lambda_{t r, k} \equiv 2\left(\alpha^{2} F\right)_{t r, k} / \omega_{k}$, partial contribution of the mode to $\lambda_{t r}$; two sets of numbers show the results of two independent runs. $\bar{\omega}_{\mathrm{ln}},\left\langle\bar{\omega}^{2}\right\rangle^{1 / 2}$ : generalized moments of $F(\omega) . \omega_{\ln },\left\langle\omega^{2}\right\rangle^{1 / 2}$ : generalized moments of $\alpha^{2} F_{t r}(\omega)$ (see text).

\begin{tabular}{lcc}
\hline \hline & $F_{k} / \omega_{k}\left(\mathrm{eV}^{-1}\right)$ & $\lambda_{t r, k}=\left(\alpha_{t r}^{2} F\right)_{k} / \omega_{k}$ \\
$\omega_{k}(\mathrm{meV})$ & $\begin{array}{c}\text { First run, } 2-300 \mathrm{~K}, H=1 \mathrm{~T} \\
\text { (second run, } 16-300 \mathrm{~K}, H=0)\end{array}$ & $\begin{array}{c}\text { First run, } 2-300 \mathrm{~K}, H=5 \mathrm{~T} \\
\text { (second run, } 7.5-300 \mathrm{~K}, H=0)\end{array}$ \\
\hline 127 & $3.37(3.40)$ & $0(0)$ \\
72.7 & $4.60(4.61)$ & $0.101(0.117)$ \\
41.6 & $4.07(4.01)$ & $0(0)$ \\
23.8 & $0.80(0.93)$ & $0(0)$ \\
13.6 & $3.37(3.21)$ & $0(0.006)$ \\
7.76 & $13.0(13.1)$ & $0.724(0.743)$ \\
4.43 & $0.83(0.93)$ & $0.180(0.186)$ \\
2.53 & $0.12(-)$ & $0.024(0)$ \\
1.45 & $0.009(-)$ & $(-)$ \\
0.83 & $0.013(-)$ & $(-)$ \\
& $\Sigma F_{k}=1.103(1.105)$ & $\lambda_{t r}=\Sigma \lambda_{t r, k}=1.03(1.05)$ \\
& $\bar{\omega}_{\ln }=20.0(20.2) \mathrm{meV}$ & $\omega_{\ln }=8.5(9.0) \mathrm{meV}$ \\
& $\left\langle\bar{\omega}^{2}\right\rangle^{1 / 2}=53.9(54.0) \mathrm{meV}$ & $\left\langle\omega^{2}\right\rangle^{1 / 2}=23.8(25.2) \mathrm{meV}$ \\
\hline \hline
\end{tabular}

A comparison with standard determinations of the PDOS is instructive. $\mathrm{YB}_{6}$ has been studied by inelastic incoherent neutron scattering on polycrystals. ${ }^{30}$ According to this unpublished work, the GDOS, i.e., the generalized PDOS weighted by the scattering cross sections of the $\mathrm{Y}$ and $\mathrm{B}$ atoms, extends to about $200 \mathrm{meV}$ and exhibits a lowfrequency peak at $\sim 10 \mathrm{meV} .{ }^{16}$ However, the integrated GDOS up to $15 \mathrm{meV}$ only contains $1.3 \%$ of the total weight, one order of magnitude below the result from the specific heat. The GDOS and the true PDOS are expected to differ in the present case owing to the different scattering cross sections of yttrium and boron. A determination of the PDOS by inelastic neutron scattering on single crystals is not available for $\mathrm{YB}_{6}$, but dispersion curves up to $\sim 60 \mathrm{meV}$ are known for the isostructural compounds $\mathrm{LaB}_{6}$ and $\mathrm{SmB}_{6} \cdot{ }^{31,32} \mathrm{In}$ both cases, it was found that the optical modes are separated from the acoustic ones by a wide gap, which can be observed around $24 \mathrm{meV}$ in the PDOS of $\mathrm{YB}_{6}$ derived from the specific heat (Fig. 9 below). It was also pointed out that the acoustic modes of $\mathrm{LaB}_{6}\left(\mathrm{SmB}_{6}\right)$, both longitudinal and transverse, are unusually flat over the major part of the Brillouin zone, due to the noninteracting vibration of the $\mathrm{La}(\mathrm{Sm})$ ion. This gives rise to a low-frequency peak at $\sim 2.5-3 \mathrm{THz}$ $(\sim 10-12 \mathrm{meV})$ in the PDOS. Although yttrium is lighter, this peak lies at $\sim 8 \mathrm{meV}$ in $\mathrm{YB}_{6}$ according to the specific heat. This softening is associated with the weaker bond due to the smaller radius of the metal ion, while the size of the boron cage undergoes little change.

High frequency modes have been studied by Raman techniques in the hexaboride series (see Ref. 33 and references therein). They are associated with "internal" modes of the boron octahedra. Their energies cannot be resolved by the deconvolution of the specific heat, but their weight is included in the highest two frequency bins.

\section{RESISTIVITY AND ELECTRON-PHONON COUPLING}

The resistivity (Fig. 7) is analyzed in a similar way. We start from the generalized Bloch-Grüneisen formula (see, e.g., Ref. 34, in particular pp. 212 and 219)

$$
\rho_{B G}(T)=\rho(0)+\frac{4 \pi m^{*}}{n e^{2}} \int_{0}^{\omega_{\max }} \alpha_{t r}^{2} F(\omega) \frac{x e^{x}}{\left(e^{x}-1\right)^{2}} d \omega,
$$

where $x \equiv \omega / T$ and $\alpha_{t r}^{2} F(\omega)$ is the electron-phonon "transport coupling function." In the restricted Bloch-Grüneisen approach, one would have $\alpha_{t r}^{2} F(\omega) \propto \omega^{4}$, and as a consequence

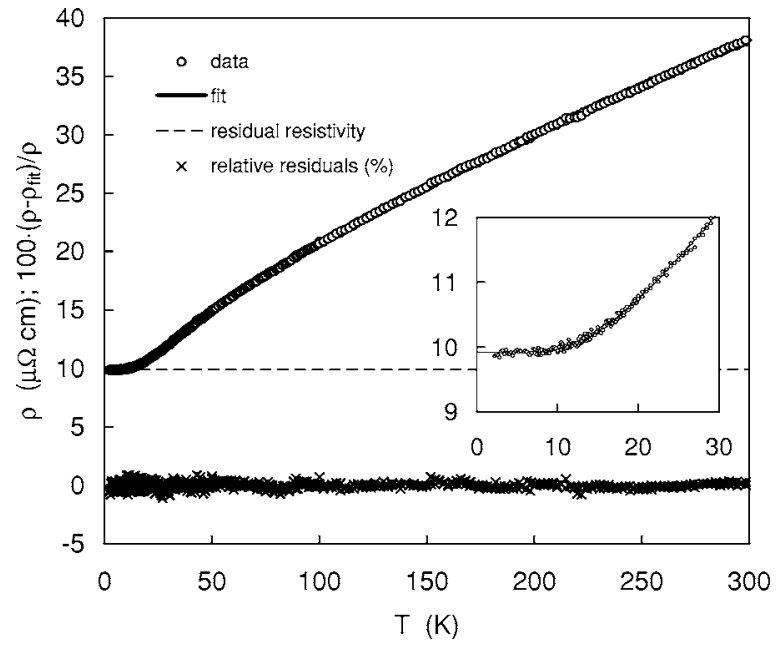

FIG. 7. Normal-state resistivity of $\mathrm{YB}_{6}$ vs the temperature. Dashed line: residual resistivity. Crosses: residuals of the fit in \%. Inset: expanded low-temperature data and polynomial fit. Superconductivity is quenched by a field of $1 \mathrm{~T}$. 
$\rho_{B G}(T)-\rho(0) \propto T^{5}$, but deviations from the Debye model, complications with phonon polarizations, and umklapp processes would not justify this simplification beyond the lowtemperature continuum limit-i.e., only a few kelvin in this case. Using a decomposition into a basis of Einstein modes similar to Eq. (5),

$$
\alpha_{t r}^{2} F(\omega)=\frac{1}{2} \sum_{k} \lambda_{t r, k} \omega_{k} \delta\left(\omega-\omega_{k}\right),
$$

we obtain the discrete version of Eq. (9):

$$
\rho_{B G}(T)=\rho(0)+\frac{2 \pi}{\varepsilon_{0} \Omega_{p k}^{2}} \sum_{t r, k} \omega_{k} \frac{x_{k} e^{x_{k}}}{\left(e^{x_{k}}-1\right)^{2}},
$$

where the fitting parameters are the dimensionless constants $\lambda_{t r, k}$. The constraint $\lambda_{t r, k} \geqslant 0$ is enforced. The residual resistivity $\rho(0)=9.9 \mu \Omega \mathrm{cm}$ is determined separately (inset of Fig. 7). The unscreened plasma frequency $\Omega_{p}$ $\equiv\left(n e^{2} / \varepsilon_{0} m^{*}\right)^{1 / 2}=5.2 \mathrm{eV}$ is taken from extensive optical spectroscopy experiments performed on the same single crystal, to be published elsewhere. ${ }^{21}$ The negative curvature of the resistivity at high temperature, a rather general phenomenon possibly related to the Mott limit, ${ }^{35,36}$ is taken into account by the empirical "parallel-resistor" formula ${ }^{37}$

$$
\frac{1}{\rho(T)}=\frac{1}{\rho_{B G}(T)+\rho(0)}+\frac{1}{\rho_{\max }} .
$$

The parameter $\rho_{\max }=73 \mu \Omega \mathrm{cm}$ is fitted simultaneously to the parameters $\lambda_{t r, k}$, using data taken in either $H=0\left(T_{c} \leqslant T\right.$ $\leqslant 300 \mathrm{~K})$ or $5 \mathrm{~T}(2.2 \leqslant T \leqslant 300 \mathrm{~K})$. These two data sets are fitted independently (rms error $\sim 0.2 \%$ ) in order to evaluate the robustness of the results (Table IV). Only a few basis modes contribute. Two low-energy modes at $\sim 8$ and $\sim 4.5 \mathrm{meV}$ clearly stand out (Fig. 9 below), in agreement with tunneling data featuring a peak in the Eliashberg function $\alpha^{2} F(\omega)$ at $8.5 \mathrm{meV}$ and a shoulder at $\sim 5 \mathrm{meV}{ }^{26}$ The electron-phonon coupling parameter relevant for transport $\lambda_{t r} \equiv 2 \int \omega^{-1} \alpha_{t r}^{2} F(\omega)$ is obtained from $\lambda_{t r}=\Sigma_{k} \lambda_{t r, k}=1.04$. Within experimental accuracy, it is equal to the electronphonon coupling parameter relevant for superconductivity obtained in the previous section, $\lambda_{e p} \equiv 2 \int \omega^{-1} \alpha^{2} F(\omega)=1.01$. This is expected for phonon-mediated superconductors, ${ }^{22,34}$ but demonstrated experimentally in the present case. Finally, the value of $\omega_{\ln }$ given by

$$
\omega_{\mathrm{ln}} \equiv \exp \left(\frac{\int \omega^{-1} \ln \omega \alpha_{\mathrm{tr}}^{2} F(\omega) d \omega}{\int \omega^{-1} \alpha_{t r}^{2} F(\omega) d \omega}\right)=\exp \left(\frac{1}{\lambda} \sum_{k} \lambda_{k} \ln \omega_{k}\right)
$$

and the alternative determination of $\omega_{\mathrm{ln}}$ obtained from the dimensionless specific heat jump in Sec. III both give the same value, $8.7 \mathrm{meV}$. Numerical results are summarized in Table IV, including the generalized second moment

$$
\left\langle\omega^{2}\right\rangle^{1 / 2} \equiv\left(\frac{\int \omega \alpha_{t r}^{2} F(\omega) d \omega}{\int \omega^{-1} \alpha_{t r}^{2} F(\omega) d \omega}\right)^{1 / 2}=\left(\frac{1}{\lambda} \sum_{k} \lambda_{k} \omega_{k}^{2}\right)^{1 / 2} .
$$

When compared with thermodynamic data, the analysis of the dc and optical conductivity therefore leads to the conclusion that superconductivity is essentially driven by a single low-energy mode (or a narrow group of modes), since $\omega_{\ln }$ is very close to the low-frequency peak of the PDOS. This conclusion, which is at odds with early electronic-structure and phonon-mode calculations, ${ }^{16}$ fully supports tunneling spectroscopy experiments. ${ }^{26} \mathrm{YB}_{6}$ is an almost ideal case of a superconductor with an Einstein PDOS. Just as in $\mathrm{ZrB}_{12}$, ${ }^{4}$ most of the electron-phonon coupling arises from the largeamplitude, low-frequency vibrations of the loosely bound metal atoms in the oversized boron cages.

More generally, it is interesting to note that the resistivity equation (11) can be reexpressed in a form that emphasizes the similarity with the specific heat [Eq. (6)]:

$$
\frac{\rho_{B G}(T)-\rho(0)}{T}=\frac{R_{0} k_{B}}{\varepsilon_{0} V_{p}^{2}} \sum_{k} \lambda_{t r, k} E\left(T / T_{k}\right),
$$

where $R_{0} \equiv h / e^{2}=25.8 \mathrm{k} \Omega$ is the quantum of resistance, $V_{p}$ the plasma frequency in volts, and $E(x) \equiv x^{2} e^{x} /\left(e^{x}-1\right)^{2}$ the normalized Einstein specific heat at a temperature $T$ due to a mode with a characteristic temperature $T_{k}$. In the case of coupling to a single mode, this reduces to

$$
\frac{\rho_{B G}(T)-\rho(0)}{C_{p h} T}=\frac{R_{0} k_{B}}{\varepsilon_{0}} \frac{\lambda_{t r}}{V_{p}^{2}}=\text { const. }
$$

This scaling of $C_{p h}$ and $\left[\rho_{B G}(T)-\rho(0)\right] / T$ is approximately obeyed in $\mathrm{ZrB}_{12}$ and $\mathrm{YB}_{6}$ due to the predominance of one soft mode. Grimvall ${ }^{34}$ noted that a similar relation holds in the high-temperature limit for any number of modes. As no large variation is expected in the plasma frequency $\Omega_{p}$ $\cong\left(e^{2} / \varepsilon_{0} m_{e} V_{\text {cell }}\right)^{1 / 2}$ of trivalent hexaborides which have one free carrier per unit cell, ${ }^{9} \mathrm{Eq}$. (16) immediately shows that $\lambda_{t r}$ in $\mathrm{LaB}_{6}$, which has a room-temperature phonon resistivity $\rho(300)-\rho(0)=8.9 \mu \Omega \mathrm{cm},{ }^{38}$ is much weaker than $\lambda_{t r}$ in $\mathrm{YB}_{6}$, for which $\rho(300)-\rho(0)=28.4 \mu \Omega \mathrm{cm}$. Indeed superconductivity has been reported to occur only below $0.1 \mathrm{~K}$ in $\mathrm{LaB}_{6}$. More information on the possibilities and limitations of the deconvolution of the resistivity to obtain the $\alpha_{t r}^{2} F(\omega)$ function may be found in the paper of Igalson et al. ${ }^{39}$

\section{THERMAL EXPANSIVITY AND ANHARMONICITY}

Thermal-expansion experiments were undertaken to give three types of information: (i) confirmation of the main features of the PDOS, (ii) evaluation of the volume dependence of phonon modes and electronic density of states, and (iii) determination of the variation of $T_{c}$ with pressure. The linear thermal expansivity $\alpha(T)$ for a cubic system is given by 


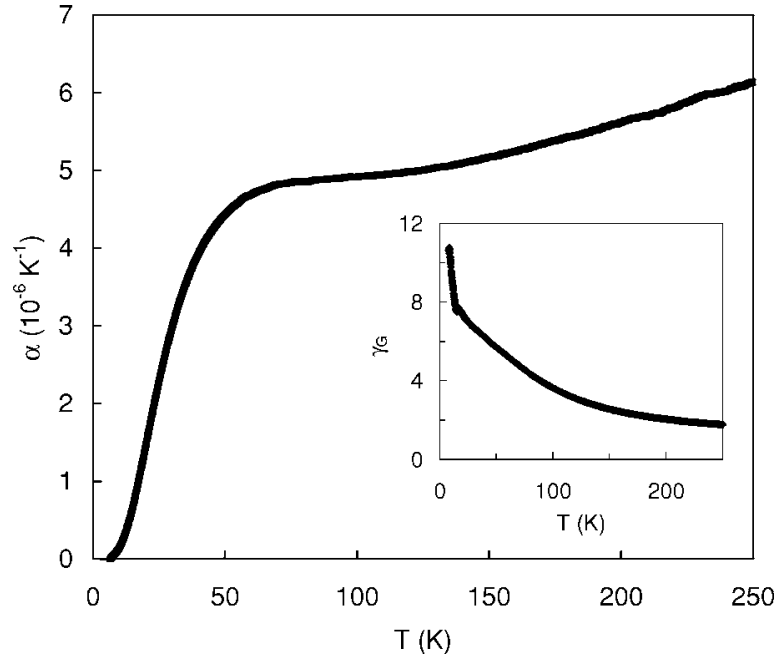

FIG. 8. Linear thermal expansivity of $\mathrm{YB}_{6}$ vs temperature. Inset: Grüneisen parameter vs temperature, assuming a bulk modulus $\kappa_{T}^{-1}=190 \mathrm{GPa}$.

$$
\alpha(T) \equiv \frac{1}{L}\left(\frac{\partial L}{\partial T}\right)_{p}=\frac{\kappa_{T}}{3}\left(\frac{\partial S}{\partial V}\right)_{T},
$$

where $\kappa_{T}$ is the isothermal compressibility. The expansivity is closely related to the specific heat at constant volume via the Grüneisen parameters (see, e.g., Ref. 34):

$$
\alpha(T)=\frac{\kappa_{T}}{3 V}\left(\gamma_{G, e l} C_{e l}+\gamma_{G, p h} C_{p h}\right),
$$

where the electronic Grüneisen parameter $\gamma_{G, e l}$ $=\partial \ln \gamma_{n} / \partial \ln V$ gives a measure of the volume dependence of the Sommerfeld constant and the phonon Grüneisen parameter $\gamma_{G, p h} \equiv-\partial \ln \omega / \partial \ln V$ represents the anharmonicity of the lattice vibrations. In this simple form, we can make use of the known components $C_{e l}(T)$ and $C_{p h}(T)$ of the specific heat in the normal state and adjust $\gamma_{G, e l}$ and $\gamma_{G, p h}$ to fit the normal-state expansivity curve $\alpha(T)$ at low temperature. As in the case of the specific heat, a plot of $\alpha / T$ versus $T^{2}$ is most suitable for displaying the results. The fitted parameters $\gamma_{G, e l}=15 \pm 3$ and $\gamma_{G, p h}=9 \pm 1$ are stable when the upper limit of the fit is varied between 50 and $120 \mathrm{~K}^{2}$. This determination of $\gamma_{G, p h}$ is representative of the lowest frequencies of the phonon spectrum; at higher temperatures, the quality of the fit degrades rapidly. With $\gamma_{G, e l}$ we determine the electronic component of the expansivity, $\quad \alpha_{e l}(T) / T=(2.6 \pm 0.5)$ $\times 10^{-9} \mathrm{~K}^{-2}$. In these evaluations the bulk modulus $\kappa_{T}^{-1}$ $=190 \mathrm{GPa}$ has been estimated from Fig. 2 of Ref. 33; the value given by band-structure calculations is $\kappa_{T}^{-1}=179 \mathrm{GPa}^{20}$

At higher temperature, the frequency dependence of the phonon Grüneisen parameter must be taken into account. Modes which are characterized by a large $\gamma_{G, p h}(\omega)$ are more heavily weighted in the thermal expansion than in the lattice specific heat. This is exemplified by the expansivity data shown in Fig. 8 over the full temperature range, to be compared with the specific heat in Fig. 5. The broad anomaly which appears around $50 \mathrm{~K}$ in Fig. 8 is evidence for a large

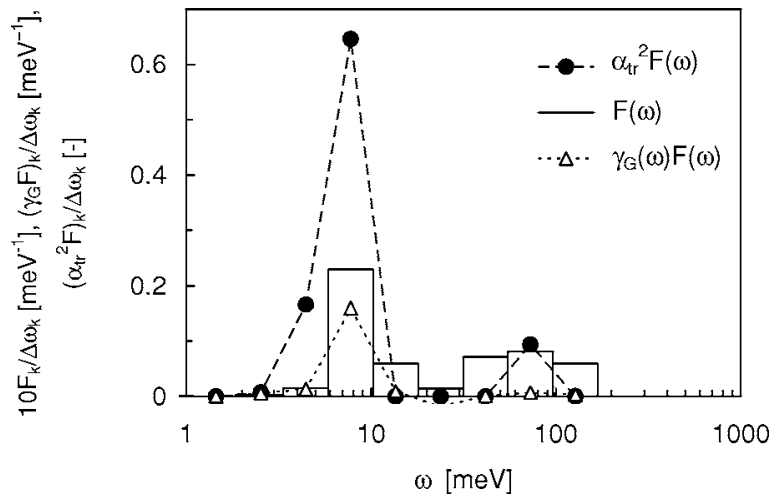

FIG. 9. Phonon density of states $F(\omega)$ deconvoluted from the specific heat, electron-phonon transport coupling function $\alpha_{t r}^{2} F(\omega)$ deconvolved from the resistivity, and spectral anharmonicity function $\gamma_{G}(\omega) F(\omega) \equiv-(\partial \ln \omega / \partial \ln V) F(\omega)$ deconvoluted from the thermal expansion. Fits are performed with $\delta$ functions $F_{k} \delta\left(\omega-\omega_{k}\right)$, $\left(\alpha_{t r}^{2} F\right)_{k} \delta\left(\omega-\omega_{k}\right)$, and $\left(\gamma_{G} F\right)_{k} \delta\left(\omega-\omega_{k}\right)$, respectively, on a basis of Einstein frequencies $\omega_{k+1}=1.75 \omega_{k}$ (see Fig. 6). In order to reflect the spectral density, the $\delta$ functions of the PDOS are represented by rectangles having a width $\Delta \omega_{k} \equiv 1.75^{1 / 2} \omega_{k}-\omega_{k} / 1.75^{1 / 2} \cong 0.57 \omega_{k}$ and a height $F_{k} / \Delta \omega_{k}$. In a similar way, the $\delta$ functions of the $\alpha_{t r}^{2} F(\omega)$ function are represented by solid circles at a height $\left(\alpha_{t r}^{2} F\right)_{k} / \Delta \omega_{k} \cong 0.88 \lambda_{k}$ and those of $\gamma_{G}(\omega) F(\omega)$ by triangles at a height $\left(\gamma_{G} F\right)_{k} / \Delta \omega_{k}$. The dashed lines are guides for the eye.

volume dependence in some low-frequency modes. In order to evaluate the energy of these modes, we fit the phonon expansivity over the full temperature range in a similar manner to the resistivity and the specific heat, using the same set of Einstein frequencies. Equation (19) below, similar to Eqs. (6) and (11), allows the parameters $\gamma_{G, k} F_{k}$ to be extracted for each frequency $\omega_{k}$ :

$$
\alpha_{p h}(T)=\alpha(T)-\alpha_{e l}(T)=\frac{N_{A v} k_{B} \kappa_{T}}{V} \sum_{k} \gamma_{G, k} F_{k} \frac{x_{k}^{2} e^{x_{k}}}{\left(e^{x_{k}}-1\right)^{2}} .
$$

The PDOS weighted by the frequency-dependent Grüneisen parameter, $\gamma_{G, p h}(\omega) F(\omega)$, is represented in Fig. 9 together with other spectra. The $8-\mathrm{meV}$ and $4.5-\mathrm{meV}$ modes are heavily weighted with $\gamma_{G, k} \cong 7$ and 9 , respectively, whereas the other modes are much less anharmonic with $\gamma_{G, k}$ values below 2. ${ }^{40}$ Similarly to $\mathrm{MgB}_{2}$ and $\mathrm{ZrB}_{12}$, the modes which give rise to a large electron-phonon coupling are anharmonic.

The pressure dependence of $T_{c}$ is obtained from the Ehrenfest relation

$$
\Delta \alpha=\frac{1}{3 V} \frac{\Delta C}{T_{c}}\left(\frac{\partial T_{c}}{\partial p}\right)_{T},
$$

where $\Delta \alpha$ and $\Delta C$ represent discontinuities of $\alpha$ and $C$ at the second-order transition. The experimentally determined step $\Delta \alpha=-(3.5 \pm 0.5) \times 10^{-8} \mathrm{~K}^{-1} \quad$ (Fig. 10) corresponds to $-0.53 \pm 0.08 \mathrm{~K} / \mathrm{GPa}$ for the initial pressure dependence of $T_{c}$. Again assuming $\kappa_{T}^{-1}=190 \mathrm{GPa}$, one obtains the fractional volume dependence of the critical temperature $\partial \ln T_{c} / \partial \ln V=14 \pm 2$. 


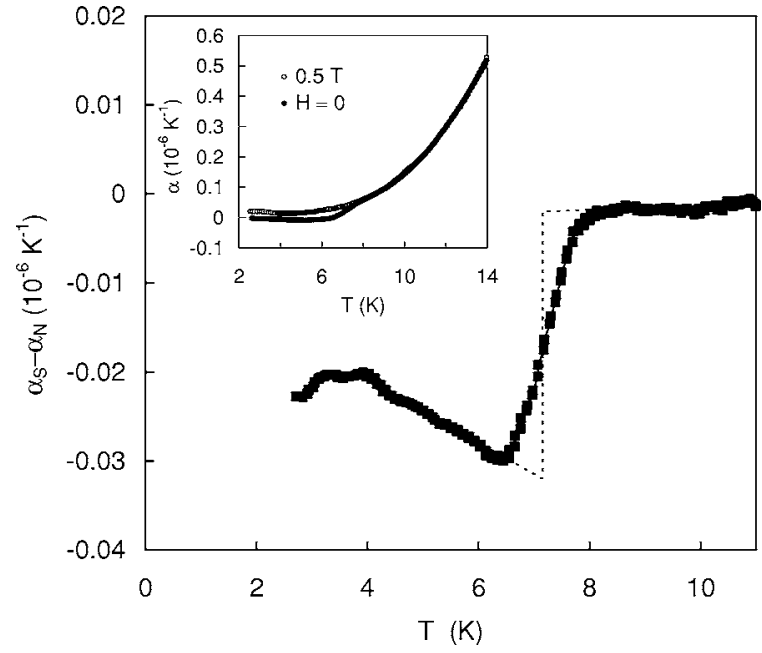

FIG. 10. Difference between the normal-state and superconducting-state linear thermal expansivity near $T_{c}$. The idealized jump is shown by a dotted line. Inset: expansivity in the normal and superconducting states.

The fractional volume dependences of the critical temperature and Sommerfeld constant are unusually large, 14 and 15 , respectively. The fact that they are nearly equal is associated with the fact that the relative jumps of the expansivity and specific heat are equal in magnitude but opposite in sign. This follows from Eqs. (18) and (20):

$$
\left(\frac{\partial \ln T_{c}}{\partial \ln V}\right)_{T}=-\frac{\Delta \alpha / \alpha_{e l}\left(T_{c}\right)}{\Delta C / C_{e l}\left(T_{c}\right)}\left(\frac{\partial \ln \gamma_{n}}{\partial \ln V}\right)_{T} .
$$

The roles of the phonon modes and the EDOS in the volume dependence still have to be elucidated. If the transition temperature is derived from a McMillan-type relation, ${ }^{41}$ by neglecting the volume dependence of the screened Coulomb repulsion parameter $\mu^{*}$ and recalling that the Eliashberg function is strongly peaked, $\alpha^{2} F(\omega) \approx \lambda \omega_{E} \delta\left(\omega-\omega_{E}\right)$, we obtain $^{34}$

$$
\frac{d \ln T_{c}}{d \ln V}=-\gamma_{G, p h}\left(\omega_{E}\right)+f\left(\lambda_{e p}, \mu^{*}\right) \frac{d \ln \lambda_{e p}}{d \ln V},
$$

where $f\left(\lambda_{e p}, \mu^{*}\right)$ is easily calculated from McMillan's equation and takes the value $\sim 1.5$ in the present case with $\lambda_{e p}$ $\cong 1, \mu^{*} \cong 0.1$. A second equation describes the fractional volume dependence of $\gamma_{n} \propto N\left(E_{F}\right)\left(1+\lambda_{e p}\right)$ :

$$
\frac{d \ln \gamma_{n}}{d \ln V}=\frac{d \ln N\left(E_{F}\right)}{d \ln V}+\frac{\lambda_{e p}}{1+\lambda_{e p}} \frac{d \ln \lambda_{e p}}{d \ln V} .
$$

From Eqs. (22) and (23), $\partial \ln N\left(E_{F}\right) / \partial \ln V=8.2 \pm 4$ and $\partial \ln \lambda_{e p} / \partial \ln V=13.7 \pm 2$. The variation of $\gamma_{n}$ with the volume is therefore due to the variation of both the EDOS and the phonon-dependent renormalization in an approximately equal ratio. ${ }^{42}$

In a final step we may write $\lambda_{e p}=\eta / M \omega_{E}^{2}$, where $\eta$ is the Hopfield electronic parameter. It follows that

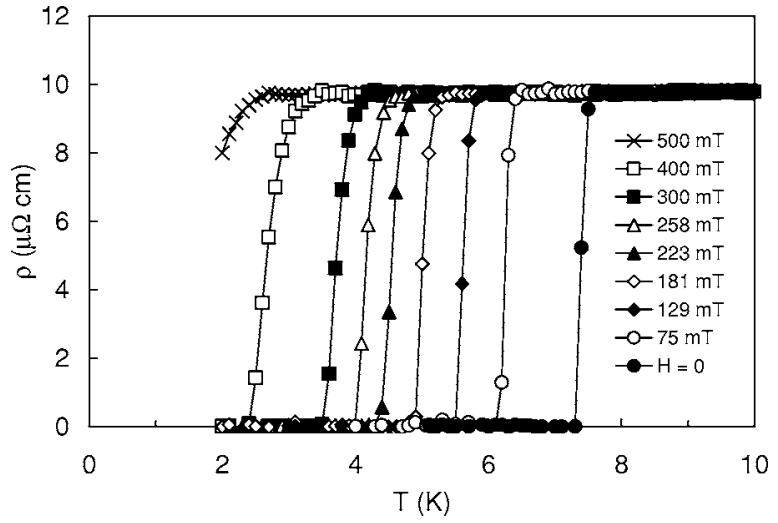

FIG. 11. Resistivity of $\mathrm{YB}_{6}$ near $T_{\mathrm{c}}$ as a function of the temperature in different magnetic fields.

$$
\frac{d \ln \lambda_{e p}}{d \ln V}=\frac{d \ln \eta}{d \ln V}+2 \gamma_{G, p h}\left(\omega_{E}\right) .
$$

The first term of the right-hand side is found to be small, $d \ln \eta / d \ln V=-0.3 \pm 3$, compared to the second one, $2 \gamma_{G, p h}\left(\omega_{E}\right)=14 \pm 2$. Therefore we may say that the large and positive values of $\partial \ln \lambda_{e p} / \partial \ln V$ and $\partial \ln T_{c} / \partial \ln V$ are essentially due to the anharmonicity of the $8-\mathrm{meV}$ mode. When the volume of the boron cages increases, the force constant which determines the frequency of the strongly coupled soft mode decreases and the latter moves closer to the favorable region $\approx 6 k_{B} T_{c}$ where the functional derivative $\delta T_{c} / \delta \alpha^{2} F(\omega)$ is largest. ${ }^{8}$

\section{MAGNETIC PHASE DIAGRAM}

The superconducting phase diagram in the $H-T$ plane was investigated by magnetoresistance, magnetization, and specific heat measurements, allowing the critical fields $H_{c 1}(T)$, $H_{c}(T), H_{c 2}(T)$, and $H_{c 3}(T)$ to be determined (Table III). The resistive transitions in fields $0-0.5 \mathrm{~T}$ are shown in Fig. 11. They are measured with both the field and current parallel to the long axis of the crystal. The extrapolation of the steepest part of the transition to $R=0$ is used to define the surface critical field $H_{c 3}(T)$, which is well separated from $H_{c 2}(T)$ as already observed in the dodecaboride $\mathrm{ZrB}_{12}{ }^{5}$

The dc magnetization at temperatures from 2 to $6 \mathrm{~K}$ is shown in Fig. 12. Our data agree with those of Kunii et al. ${ }^{43}$ The shape is typical of a type-II superconductor. The sharp minimum at the border of the Meissner and mixed-state regions defines $H_{c 1}(T)$, and the break in the slope between the mixed-state and normal-state regions defines $H_{c 2}(T)$. It is remarkable that no anomaly can be detected in the magnetization at $H_{c 3}(T)$ where the resistance vanishes (Fig. 13), thus confirming the superficial nature of the third critical field. No anomaly is detected in the specific heat either at $H_{c 3}(T)$. Note that the scale of Fig. 13 is enlarged by a factor of 5000 with respect to that of Fig. 12. Although on this scale the transition at $H_{c 2}$ appears to be rounded, $H_{c 2}$ nevertheless remains well defined using an extrapolation of the linear parts from above and below the transition, as expected in the GinzburgLandau regime (Fig. 13, inset). 


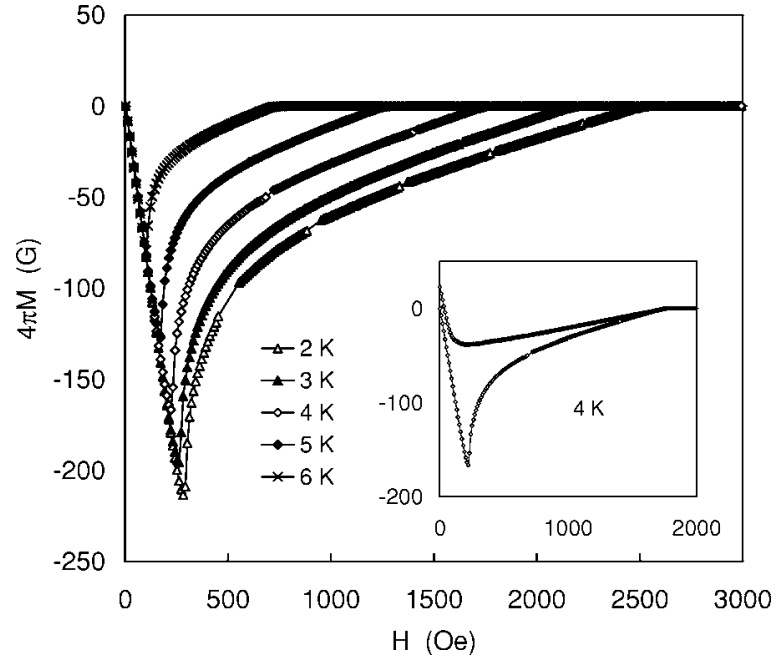

FIG. 12. Magnetization of $\mathrm{YB}_{6}$ as a function of the magnetic field for different temperatures, virgin curves with increasing field $\left(M\right.$ in emu $/ \mathrm{cm}^{3}, 4 \pi M$ in $\left.\mathrm{G}\right)$. Inset: sample hysteresis curve at $4 \mathrm{~K}$ (similar curves are obtained from 2 to $6 \mathrm{~K}$ ).

Finally the specific heat was measured in several fields from 0 to $1 \mathrm{~T}$. These measurements give an independent bulk determination of $H_{c 2}(T)$. The criterion used here is the midpoint of the step at the transition (Fig. 14). No anomaly is seen at $H_{c 1}(T)$, thus establishing that $\mathrm{YB}_{6}$ is a type-II/2 superconductor, unlike $\mathrm{ZrB}_{12}$ which was of type-II/ $1 .{ }^{5}$ Note that in Fig. 14 only the electronic part $C_{e} / T$ of the total specific heat is shown. The rise of $C_{e}(H) / T$ with respect to the zero-field curve $C_{e}(0) / T$, shown in Fig. 15, is due to the contribution of quasi normal vortex cores and the excitations of the vortex lattice. The trends observed in the data suggest

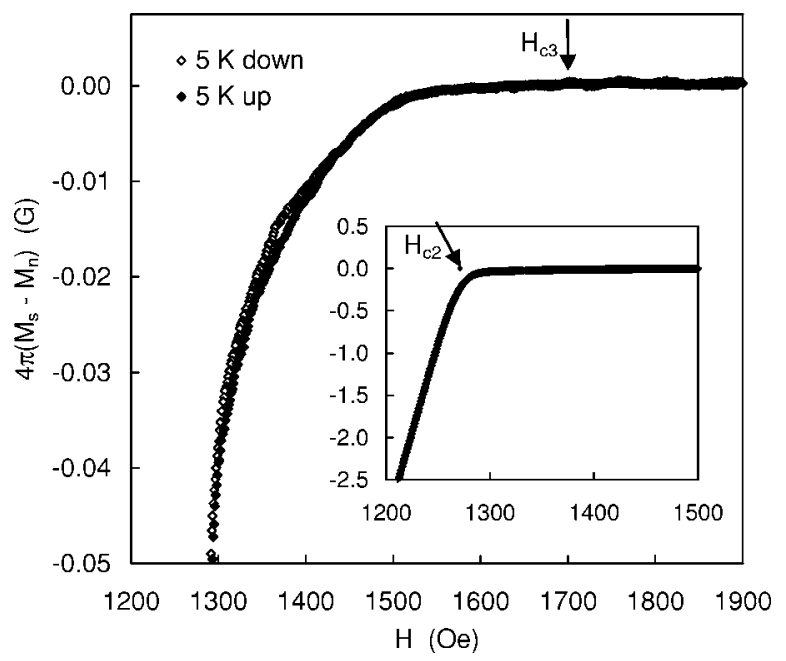

FIG. 13. Detail of the previous plot at $T=5 \mathrm{~K}$, expanded 100 times in the inset and 5000 times in the main frame. The normalstate magnetization $M_{\mathrm{n}}$ has been subtracted. Note the absence of any measurable diamagnetism at $H_{\mathrm{c} 3}=1700 \mathrm{G}$ where the resistance drops to zero. Fluctuation diamagnetism sets in smoothly near $1500 \mathrm{G}$. The upper critical field $H_{\mathrm{c} 2}=1270 \mathrm{G}$ defined by the intersection of the extrapolated linear sections in the inset coincides with the position of the specific heat jump.

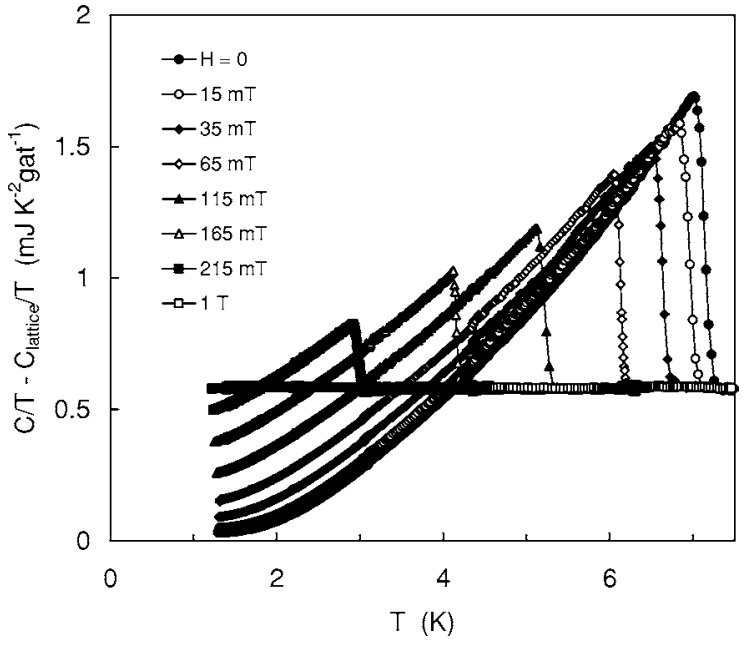

FIG. 14. Electronic specific heat of $\mathrm{YB}_{6}$ divided by the temperature vs the temperature for different magnetic fields.

that the model of Caroli et al. ${ }^{44,45}$-i.e. $C_{e}(H) / T$ $=\gamma_{n} H / H_{c 2}(0)$ - would be obeyed at temperatures below $\sim 0.1 T_{c}$.

The above information is summarized in the phase diagram, Fig. 16. The Maki parameter $\kappa_{1}(T)$ $\equiv 2^{-1 / 2} H_{c 2}(T) / H_{c}(T)$ shown in the inset extrapolates to $\kappa_{1}\left(T_{c}\right) \equiv \kappa \cong 3$ and increases at low temperature as usual. Note that $\kappa$ is too low for the approximate Ginzburg-Landau relation $H_{c 1}(T) \cong 2^{-1 / 2} H_{c}(T) \ln \kappa_{1} / \kappa_{1}$ to hold. Instead we use $\kappa_{1}(T)$ to recalculate $H_{c 1}(T)$ according to the numerical work of Harden and $\operatorname{Arp}^{46}$ (lower dashed line in Fig. 16). These recalculated values nevertheless underestimate $H_{c 1}(T)$ taken from magnetization curves by $10 \%-20 \%$.

The critical field $H_{c 3}(T)$, which describes the persistence of superconductivity over a layer of thickness comparable to the coherence length $\xi$ when the field is applied parallel to a flat surface, should ideally be $1.695 H_{c 2}(0) .{ }^{47,48}$ Indeed, at

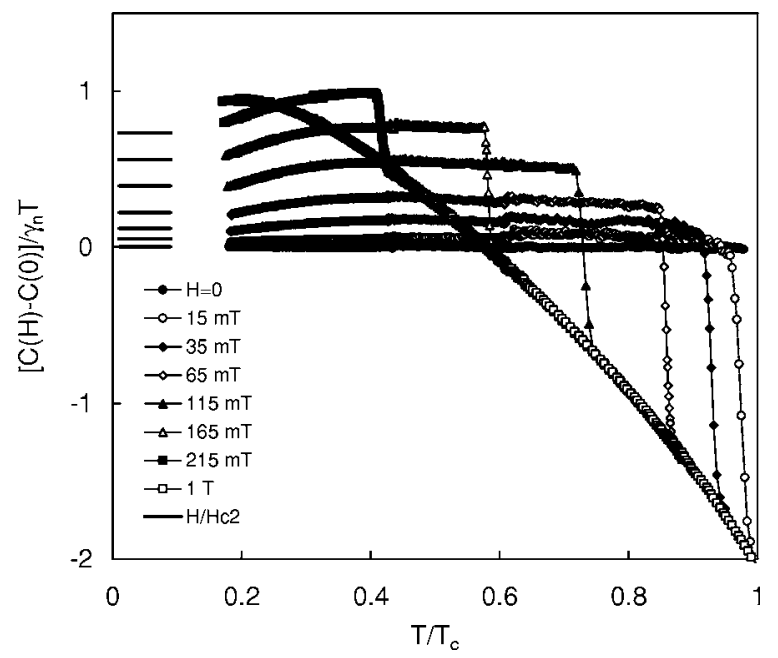

FIG. 15. Specific heat difference $[C(H, T)-C(0, T)] / T$ normalized by the Sommerfeld constant vs $T / T_{c}$ in different magnetic fields. The value calculated within the Caroli-Matricon-de Gennes approximation is shown by solid lines at $T \rightarrow 0$. 


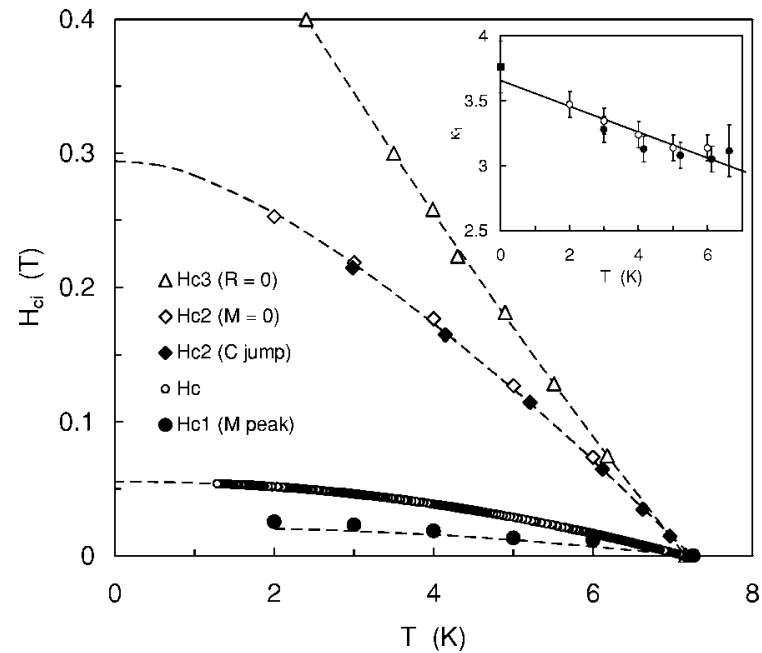

FIG. 16. Phase diagram of $\mathrm{YB}_{6}$ in the $H-T$ plane. From top to bottom: third critical field $H_{\mathrm{c} 3}(T)$ defined by zero resistance for $H$ parallel to the current and surface, second upper critical field $H_{\mathrm{c} 2}(T)$ given by the position of the specific heat jump (closed diamonds) and the knee of the magnetization (open diamonds), thermodynamic critical field $H_{\mathrm{c}}(T)$ obtained by integration of the specific heat $C / T$ (open circles), and lower critical field $H_{\mathrm{c} 1}(T)$ given by the position of the sharp minimum of the magnetization (solid circles). All lines are polynomial fits to the data, except for the $H_{\mathrm{c} 1}(T)$ line, which is recalculated based on $H_{\mathrm{c}}(T)$ and the Maki parameter $\kappa_{1}(T)$ (see text). Inset: variation of $\kappa_{1}$ with the temperature.

$2.4 \mathrm{~K}$ [the lowest temperature at which $H_{c 3}(T)$ could be measured], $H_{c 3}(T) / H_{c 2}(T)=1.66$. However, while $H_{c 2}(T)$ has a negative curvature which fits well with the WerthamerHelfand-Hohenberg (WHH) theory, ${ }^{49} H_{c 3}(T)$ has a small but positive curvature. Using a polynomial fit, $H_{c 3}(T)$ extrapolates to $\sim 2.2 H_{c 2}(0)$ at $T=0$ (Fig. 16). A qualitatively similar behavior was found in $\mathrm{ZrB}_{12} .{ }^{5}$ This exceedingly large ratio may be explained here by a decrease in the mean free path with respect to the bulk over a thin layer at the surface. An increase of the gap at the surface with respect to the bulk volume [as found in $\mathrm{ZrB}_{12}$ (Refs. 4, 5, 50, and 51)] is excluded in $\mathrm{YB}_{6}$, since in this case tunneling and specific heat determinations agree on the gap value.

\section{COMPOSITIONAL DEPENDENCE}

A broad range of superconducting critical temperatures, $1.5 \leqslant T_{c} \leqslant 8.4 \mathrm{~K}$, has been reported in the literature depending on the conditions of preparation. ${ }^{9}$ In our preliminary work, $T_{c}$ onsets from 6.5 to $7.6 \mathrm{~K}$ were obtained. Energydispersive x-ray diffraction (EDX) revealed a correlation between $T_{c}$ and the $\mathrm{Y} / \mathrm{B}$ ratio, the higher $T_{c}$ corresponding to a smaller boron concentration $\left[T_{c}\left(\mathrm{YB}_{6+x}\right) \cong 6.25\right.$ $-4.3 x \pm 0.25 \mathrm{~K}]$. Literature data confirm this tendency. ${ }^{9}$ The lattice constant was found to be almost insensitive to the $\mathrm{Y} / \mathrm{B}$ ratio, changing from $4.1002(5) \AA$ for $\mathrm{YB}_{5.7}$ with $T_{c}$ $=7.6 \mathrm{~K}$ to $4.1000(5) \AA$ for $\mathrm{YB}_{5.9}$ with $T_{c}=6.6 \mathrm{~K}$. These values are in agreement with published data. ${ }^{9}$ The possible influence of strains on $T_{c}$ cannot be excluded: the high value $T_{c}=8.4 \mathrm{~K}$ reported by Fisk et $a l^{3}{ }^{3}$ was obtained by splat-
TABLE V. Parameters of the fit $H_{c}(T) / H_{c}(0)=1-\left(T / T_{c}\right)^{2}$ of the magnetization data shown in Fig. 17 for three $\mathrm{YB}_{6+x}$ crystals: $T_{c}$, critical temperature; $H_{c}(0)$, thermodynamic critical field at zero temperature. $\gamma_{n}$, Sommerfeld constant estimated assuming $\gamma_{n} T_{c}^{2} / H_{c}^{2}(0)=$ const; RRR, residual resistivity ratio. The resistance of the sample with $T_{c}=7.4 \mathrm{~K}$ [temperature at which $H_{c}(T) \rightarrow 0$ ] vanishes at $7.6 \mathrm{~K}$.

\begin{tabular}{llll}
\hline \hline$T_{c}(\mathrm{~K})$ & 6.5 & 7.2 & 7.4 \\
$H_{c}(0)(\mathrm{mT})$ & 48 & 58 & 61 \\
$\gamma_{n}\left(\mathrm{~mJ} \mathrm{~K}^{-2} \mathrm{gat}^{-1}\right)$ & 0.50 & 0.58 (reference) & 0.62 \\
$\mathrm{RRR}$ & 3.05 & 3.87 & 4.58 \\
\hline \hline
\end{tabular}

cooling of arc-melted samples with nominal composition $\mathrm{YB}_{6}$. We tried to "anneal out" residual strains in different samples by a $36-\mathrm{h}$ heat treatment at $1600{ }^{\circ} \mathrm{C}$ in 100 bars argon pressure, followed by slow cooling. The $T_{c}$ onset of low- $T_{c}$ samples $(6.5 \mathrm{~K})$ did not change; that of high- $T_{c}$ samples $(7.6-7.8 \mathrm{~K})$ decreased by about half a kelvin. In all cases the main effect was a broadening of the transition by $2-3 \mathrm{~K}$. While such experiments tend to support the idea that strains increase $T_{c}$, the effect of losses during the heat treatment cannot be excluded.

The highest $T_{c}$ is obtained for a $\mathrm{B} / \mathrm{Y}$ ratio below 6 . In this sense the reference crystal with $T_{c}=7.2 \mathrm{~K}$ studied in the previous sections is not ideal because of the presence of boron vacancies. Therefore we briefly studied two other crystals with $T_{c}^{\text {onset }}=6.5 \mathrm{~K}$ and $7.6 \mathrm{~K}$. Their resistivity was only found to differ from that of the reference sample by the residual term, while the temperature-dependent part remained unchanged (Table V). Magnetization curves were measured with increasing field and integrated to get the thermodynamic critical field $H_{c}(T)$ (Fig. 17, Table V). Irreversibility may introduce some error by delaying the entry of vortices, leading to an overestimation of $H_{c}(T)$. However, we found that the magnetic determination of $H_{c}(T)$ was only $4 \%$ above that obtained from the specific heat for the reference sample, showing that the branch with increasing field is close to equilibrium. The net result is that $T_{c}$ is positively correlated with $H_{c}(0), \gamma_{n}$, and therefore the EDOS, as is the case for most

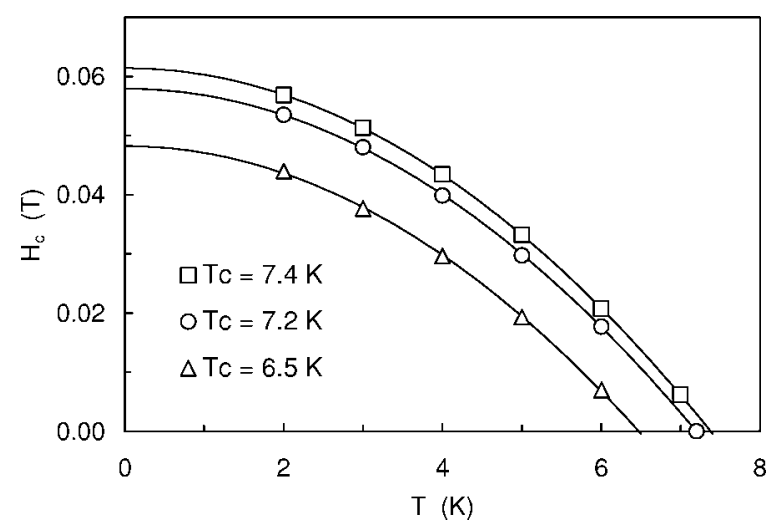

FIG. 17. Thermodynamic critical field $H_{\mathrm{c}}(T)$ of boron-rich (lower- $T_{\mathrm{c}}$ ) and boron-deficient (higher- $T_{\mathrm{c}}$ ) samples. In this plot, $H_{\mathrm{c}}(T)$ is obtained by integration of the magnetization curves. 
BCS superconductors. The change in the EDOS is confirmed by the dc magnetic susceptibility. The curve for the sample with $T_{c}=6.5 \mathrm{~K}$ is similar to that shown in Fig. 2 and described by the parameters $\chi(0)=-16.8 \times 10^{-6}, \quad a=4.0$ $\times 10^{-11} \mathrm{~K}^{-2}$, and $\mathrm{C}_{\text {Curie }}=6.5 \times 10^{-4} \mathrm{~K}$ (see Sec. II). Compared to the reference sample, the susceptibility is more diamagnetic, which is attributed to a smaller Pauli contribution. The Curie term is equivalent to $120 \mathrm{ppm} \mathrm{Yb}^{3+}$.

\section{CONCLUSION}

Specific heat, resistivity, and thermal expansion experiments performed on high quality single crystals have been used to characterize $\mathrm{YB}_{6}$. This superconductor has a low density of states at the Fermi level. Some sample dependence of $T_{c}$ can be traced to the variation of the $\mathrm{B} / \mathrm{Y}$ ratio, which in turn influences the EDOS. The specific heat in the superconducting state is typical of a single-band, isotropic BCS superconductor; however, the electron-phonon interaction is much stronger than for the other superconducting borides, in particular $\mathrm{ZrB}_{12}$ and $\mathrm{MgB}_{2}$. A common feature of these borides is nonuniform coupling to selected phonon modes. Whereas the strongly coupled modes lie at high energy $\sim 60 \mathrm{meV}$ for $\mathrm{MgB}_{2},{ }^{52}$ they lie at low energy $\sim 15 \mathrm{meV}$ for $\mathrm{ZrB}_{12}$ (Ref. 4 ) and $\sim 8 \mathrm{meV}$ for $\mathrm{YB}_{6}$, which partly explains their relatively low $T_{c}$. Similarly to $\mathrm{LaB}_{6},{ }^{29}$ these lowfrequency modes are associated with the vibration of $\mathrm{Y}$ or $\mathrm{Zr}$ atoms loosely bound in oversized boron cages. The reason for the lower characteristic frequency in $\mathrm{YB}_{6}$ compared to $\mathrm{ZrB}_{12}$ is neither to be found in the mass of the metal ions nor in the coordination number which is 24 in both cases. It is rather to be associated with the longer metal to boron bond, $3.03 \AA$ in $\mathrm{YB}_{6}$ (Ref. 53) instead of $2.76 \AA$ in $\mathrm{ZrB}_{12}$ (Ref. 54). This longer distance leads to a weaker force constant and larger vibrational amplitude, which in turn favors superconductivity. The thermal expansion indeed indicates that $T_{c}$ will decrease with pressure and that these modes are strongly anharmonic. As for magnetic properties, $\mathrm{YB}_{6}$ is a type-II superconductor with $\kappa \cong 3$ and clearly shows a third upper critical field at the surface, a rather rare occurrence.

Owing to the low characteristic energy of the phonons which mediate superconducting pairing, $\mathrm{YB}_{6}$ has been found to be an almost ideal system where, on the one hand, specific heat can substitute for inelastic neutron scattering (which is plagued by the absorption of ${ }^{10} \mathrm{~B}$ ) to study the PDOS and, on the other hand, resistivity can be substituted for tunnelling to study the electron-phonon coupling function. The spectral resolution of these procedures is limited and only makes sense on a logarithmic energy scale; nevertheless, this technique is found to be reproducible and able to give remarkably consistent values of $\lambda_{e p}$, allowing significant comparisons to be made between borides.

\section{ACKNOWLEDGMENTS}

Stimulating discussions with J. Teyssier and J. Geerk are gratefully acknowledged. We thank E. Giannini, P. Lezza, B. Revaz, and A. Naula for their help in x-ray diffraction, EDX, and metallurgy. This work was supported by the National Science Foundation through the National Centre of Competence in Research "Materials with Novel Electronic Properties-MaNEP" and INTAS Project No. 03-51-3036.
*Corresponding author. Electronic address:

Rolf.Lortz@physics.unige.ch

†Deceased.

${ }^{1}$ J. Nagamatsu, N. Nakagawa, T. Muranaka, Y. Zenitani, and J. Akimitsu, Nature (London) 410, 63 (2001).

${ }^{2}$ J. Akimitsu, in Symposium on Transition Metal Oxides, Sendai, 2001 (unpublished).

${ }^{3}$ Z. Fisk, P. H. Schmidt, and L. D. Longinotti, Mater. Res. Bull. 11, 1019 (1976).

${ }^{4}$ R. Lortz, Y. Wang, S. Abe, C. Meingast, Y. Paderno, V. Filippov, and A. Junod, Phys. Rev. B 72, 024547 (2005).

${ }^{5}$ Y. Wang, R. Lortz, Y. Paderno, V. Filippov, S. Abe, U. Tutsch, and A. Junod, Phys. Rev. B 72, 024548 (2005).

${ }^{6}$ Type-II/1 superconductivity is characterized by a first-order transition at $H_{\mathrm{c} 1}$, whereas type-II/2 has a second-order transition at $H_{\mathrm{c} 1}$ [J. Auer and H. Ullmaier, Phys. Rev. B 7, 136 (1973)].

${ }^{7}$ B. T. Matthias, T. H. Geballe, K. Andres, E. Corenzwit, G. W. Hull, and J. P. Maita, Science 159, 530 (1968).

${ }^{8}$ J. P. Carbotte, Rev. Mod. Phys. 62, 1027 (1990).

${ }^{9}$ Compounds with Boron: System Number 39, edited by H. Bergman et al., Vol. C11a of Gmelin Handbook of Inorganic Chemistry. Sc, Y, La-Lu Rare Earth Elements (Springer-Verlag, Berlin, 1990).

${ }^{10}$ T. B. Massalski, Binary Alloy Phase Diagrams Materials (ASM
International, Materials Park, OH, 1990).

${ }^{11}$ S. Otani, M. M. Korsukova, T. Mitsuhashi, and N. Kieda, J. Cryst. Growth 217, 378 (2000).

${ }^{12}$ A. Junod, in Studies of High Temperature Superconductors, edited by A. Narlikar (Nova Science, Commack, NY, 1996), Vol. 19, p. 1.

${ }^{13}$ C. Meingast, B. Blank, H. Bürkle, B. Obst, T. Wolf, H. Wühl, V. Selvamanickam, and K. Salama, Phys. Rev. B 41, 11299 (1990).

${ }^{14}$ R. Pott and R. Schefzyk, J. Phys. E 16, 444 (1983).

${ }^{15}$ P. F. Walch, D. E. Ellis, and F. M. Mueller, Phys. Rev. B 15, 1859 (1977).

${ }^{16}$ G. Schell, H. Winter, H. Rietschel, and F. Gompf, Phys. Rev. B 25, 1589 (1982).

${ }^{17}$ Y. Imai, M. Mukaida, M. Ueda, and A. Watanabe, Intermetallics 9, 721 (2001).

${ }^{18}$ I. R. Shein, S. V. Okatov, N. I. Medvedeva and A. L. A. LIvanovskii, cond-mat/0202015 (unpublished).

${ }^{19}$ B. Jäger, S. Paluch, W. Wolf, P. Herzig, O. J. Zogal, N. Shitsevalova, and Y. Paderno, J. Alloys Compd. 383, 2004 (2004).

${ }^{20}$ A. E. Baranovski, G. E. Grechnev, T. V. Ignatova, I. G. Kolobov, A. V. Logosha, Y. B. Paderno, N. Y. Shitsevalova, I. V. Svechkarev, and V. B. Filippov (unpublished).

${ }^{21}$ J. Teyssier, A. Kuzmenko, D. v. d. Marel, R. Lortz, A. Junod, V. Filippov, and N. Shitsevalova, in Proceedings of the MSM Con- 
ference, Agadir, 2005 [Phys. Status Solidi C (to be published)].

${ }^{22}$ P. B. Allen and R. C. Dynes, Phys. Rev. B 12, 905 (1975).

${ }^{23}$ A. Junod, J.-L. Jorda, and J. Muller, J. Low Temp. Phys. 62, 301 (1986).

${ }^{24}$ J. M. Coombes and J. P. Carbotte, J. Low Temp. Phys. 74, 491 (1989).

${ }^{25}$ H. Padamsee, J. E. Neighbor, and C. A. Shiffman, J. Low Temp. Phys. 12, 387 (1973).

${ }^{26}$ R. Schneider, J. Geerk, and H. Rietschel, Europhys. Lett. 4, 845 (1987).

${ }^{27}$ H. Chi and J. P. Carbotte, Phys. Rev. B 49, 6143 (1994).

${ }^{28}$ E. S. R. Gopal, Specific Heats at Low Temperatures (Heywood Books, London, 1966).

${ }^{29}$ D. Mandrus, B. C. Sales, and R. Jin, Phys. Rev. B 64, 012302 (2001).

${ }^{30} \mathrm{~B}$. Renker (private communication).

${ }^{31}$ H. G. Smith, G. Dolling, S. Kunii, M. Kasaya, B. Liu, T. Takegahara, T. Kasuya, and T. Goto, Solid State Commun. 53, 15 (1985).

${ }^{32}$ P. A. Alekseev, A. S. Ivanov, B. Dorner, H. Schober, K. A. Kikoin, A. S. Mishchenko, V. N. Lazukov, A. S. Konovalova, Y. B. Paderno, A. Y. Rumyantsev, and I. P. Sadikov, Europhys. Lett. 10, 457 (1989).

${ }^{33}$ E. Zirngiebl, S. Blumenröder, R. Mock, and G. Güntherodt, J. Magn. Magn. Mater. 54-57, 359 (1986).

${ }^{34} \mathrm{G}$. Grimvall, The Electron-Phonon Interaction in Metals (NorthHolland, Amsterdam, 1981).

${ }^{35}$ N. F. Mott, Adv. Phys. 16, 49 (1967).

${ }^{36}$ N. F. Mott, Rev. Mod. Phys. 50, 203 (1978).

${ }^{37}$ H. Wiesmann, M. Gurvitch, H. Lutz, A. Ghosh, B. Schwarz, M. Strongin, P. B. Allen, and J. W. Halley, Phys. Rev. Lett. 38, 782 (1977).

${ }^{38}$ T. Tanaka, T. Akahane, E. Bannai, S. Kawai, N. Tsuda, and Y. Ishizawa, J. Phys. C 9, 1235 (1976).

${ }^{39}$ J. Igalson, A. J. Pindor, and L. Sniadower, J. Phys. F: Met. Phys.
11, 995 (1981).

${ }^{40}$ A large negative value of the Grüneisen parameter (-13) is found in the PDOS gap around $24 \mathrm{meV}$. This value is not considered to be significant as it results from the quotient of two small quantities.

${ }^{41}$ W. L. McMillan, Phys. Rev. 167, 331 (1968).

${ }^{42}$ Band-structure calculations do not predict any significant volume dependence of the density of states at the Fermi level at the stoichiometric composition $\mathrm{B} / \mathrm{Zr}=6$. The boron deficiency of the crystal under study may play a role (see Sec. VIII) [J. Teyssier (private communication)].

${ }^{43}$ S. Kunii, T. Kasuya, K. Kadowaki, M. Date, and S. B. Woods, Solid State Commun. 52, 659 (1984).

${ }^{44}$ C. Caroli, P. G. de Gennes, and J. Matricon, Phys. Lett. 9, 307 (1964).

${ }^{45}$ C. Caroli and J. Matricon, Phys. Kondens. Mater. 3, 380 (1965).

${ }^{46}$ J. L. Harden and V. Arp, Cryogenics 3, 105 (1963).

${ }^{47}$ D. Saint-James and P. G. d. Gennes, Phys. Lett. 7, 306 (1963).

${ }^{48}$ D. K. Finnemore, T. F. Stromberg, and C. A. Swenson, Phys. Rev. 149, 231 (1966)

${ }^{49}$ N. R. Werthamer, E. Helfand, and P. C. Hohenberg, Phys. Rev. 147, 295 (1966).

${ }^{50}$ M. I. Tsindlekht, G. I. Leviev, I. Asulin, A. Sharoni, O. Millo, I. Felner, Y. B. Paderno, V. B. Filippov, and M. A. Belogolovskii, Phys. Rev. B 69, 212508 (2004).

${ }^{51}$ D. Daghero, R. S. Gonnelli, G. A. Ummarino, A. Calzolari, V. Dellarocca, V. A. Stepanov, V. B. Filippov, and Y. B. Paderno, Supercond. Sci. Technol. 17, S250 (2004).

${ }^{52}$ J. Geerk, R. Schneider, G. Linker, A. G. Zaitsev, R. Heid, K.-P. Bohnen, and H. v. Löhneysen, Phys. Rev. Lett. 94, 227005 (2005).

${ }^{53}$ P. Blum and F. Bertaut, Acta Crystallogr. 7, 81 (1954).

${ }^{54}$ B. Post and F. W. Glaser, J. Metals Trans. AIME 631 (1952).

${ }^{55}$ I. R. Shein (private communication). 